\title{
Synthesis, Structural Elucidation, and Antibacterial Evaluation of Some New Molecules Derived from Coumarin, 1,3,4-Oxadiazole, and Acetamide
}

\author{
Shahid Rasool, ${ }^{1}$ Aziz-ur-Rehman, ${ }^{1}$ Muhammad Athar Abbasi, ${ }^{1}$ Sabahat Zahra Siddiqui, ${ }^{1}$ \\ Syed Adnan Ali Shah, ${ }^{2,3}$ Sidra Hassan, ${ }^{4}$ and Irshad Ahmad ${ }^{4}$ \\ ${ }^{1}$ Department of Chemistry, Government College University, Lahore 54000, Pakistan \\ ${ }^{2}$ Faculty of Pharmacy, University Technology MARA, Puncak Alam Campus, 42300 Bandar Puncak Alam, \\ Selangor Darul Ehsan, Malaysia \\ ${ }^{3}$ Atta-ur-Rahman Institute for Natural Products Discovery (AuRIns), Level 9, FF3, University Technology MARA, \\ Puncak Alam Campus, 42300 Bandar Puncak Alam, Selangor Darul Ehsan, Malaysia \\ ${ }^{4}$ Department of Pharmacy, The Islamia University of Bahawalpur, Bahawalpur 63100, Pakistan
}

Correspondence should be addressed to Aziz-ur-Rehman; azizryk@yahoo.com

Received 30 April 2016; Accepted 25 July 2016

Academic Editor: Jonathan White

Copyright (C) 2016 Shahid Rasool et al. This is an open access article distributed under the Creative Commons Attribution License, which permits unrestricted use, distribution, and reproduction in any medium, provided the original work is properly cited.

Because of the reported biological activities of coumarin, 1,3,4-oxadiazole, and acetamides, some new compounds incorporating these moieties were synthesized and evaluated for their biological potential against Gram-positive and Gram-negative bacteria. In the present work, 4-chlororesorcinol (1) and ethyl acetoacetate (2) were mixed in a strong acidic medium to synthesize 6chloro-7-hydroxy-4-methyl-2-oxo- $2 \mathrm{H}$-chromene (3) which was subjected to the intermolecular cyclization after consecutive three steps to synthesize 5-\{[(6-chloro-4-methyl-2-oxo-2H-chromen-7-yl)oxy]methyl $\}$-1,3,4-oxadiazol-2-thiol (6). A series of acetamoyl electrophiles, 8a-o, were synthesized from aralkyl/alkyl/aryl amines, $7 \mathbf{a}-\mathbf{o}$, in an aqueous basic medium. The final compounds, $\mathbf{9 a}-\mathbf{o}$, were synthesized by the reaction of compounds $\mathbf{6}$ and $\mathbf{8 a}-\mathbf{o}$ in $\mathrm{DMF} / \mathrm{NaH}$. The synthesized compounds were structurally elucidated by spectral data analysis of IR, ${ }^{1} \mathrm{H}-\mathrm{NMR}$, and EIMS. The most of the synthesized compounds remained moderate to excellent antibacterial agents. The molecules, $\mathbf{9 e}, \mathbf{9 j}$, and $\mathbf{9 k}$, were the most efficient ones against all the five bacterial strains taken into account.

\section{Introduction}

Coumarin is a naturally occurring heterocyclic class of compounds $[1-3]$. The compounds of this class were used by ancient Egyptians as drug [4]. The field of agriculture has also found the applications of these molecules $[4,5]$. The different derivatives of various coumarins have been introduced synthetically and also by plant extraction to possess a number of biological activities including antibacterial, antioxidant, and anti-inflammatory activities [6-9]. The heterocyclic moiety, 1,3,4-oxadiazole, and its various 2,5-disubstituted products are also known to be biologically active compounds [10-12].
The presence of amide functionality is also known to boost up the potential of bioactive compounds including antifungal, anti-inflammatory, anticancer, antibacterial, and antioxidant activities [13-16].

The increased resistance by microbes against the existing drugs is the key point for search of new drug molecules [17, 18]. In continuation of our previous work on $O$-substituted derivatives [19] and notable bioactivity of ether derivatives of coumarin $[6,9,20]$ this prompted us to incorporate coumarin with 1,3,4-oxadiazole and acetamides to evaluate their antibacterial activity. 


\section{Experimental}

2.1. Material and Methods. 4-Chlororesorcinol, ethyl acetoacetate, ethyl 2-bromoacetate, hydrated hydrazine, carbon disulfide, aralkyl/alkyl/aryl amines, and 2-bromoacetyl bromide were purchased from Merck, Riedel-de Haen, Aldrich, and Alfa Aesar through local suppliers along with analytical grade solvents. The Jasco-320-A spectrophotometer was used to record IR spectra by $\mathrm{KBr}$ pellet method. The Bruker spectrometers at 125 and $400 \mathrm{MHz}$ were used to record the ${ }^{13} \mathrm{C}$ and ${ }^{1} \mathrm{H}$ NMR spectra in $\mathrm{CDCl}_{3}$, respectively. The JMS-HX-110 spectrometer was used to record EIMS spectra. Silica plates coated on alumina were used for thin layer chromatography (TLC), run in mobile phase of $n$-hexane and ethyl acetate and observed under $\mathrm{UV}_{254}$. Griffin-George apparatus was used to record the melting points in open capillary tubes which were uncorrected.

2.2. Synthesis of 6-Chloro-7-hydroxy-4-methyl-2-oxo-2Hchromene (3). 4-Chlororesorcinol (0.05 mol; 1) was dissolved in ethyl acetoacetate $(0.05 \mathrm{~mol} ; 2)$ on heating in an iodine flask $(500 \mathrm{~mL})$. Then concentrated $\mathrm{H}_{2} \mathrm{SO}_{4}(25 \mathrm{~mL})$ was added on continuous shaking at low temperature. The mixture was aged for 12-16 hours. Excess cold distilled water was added to precipitate the title compound which was separated through filtration, washed by distilled water, and dried.

2.3. Synthesis of Ethyl 2-[(6-Chloro-4-methyl-2-oxo-2Hchromen-7-yl)oxy]acetate (4). Compound 3 (0.045 mol) was dissolved in DMF $(25 \mathrm{~mL})$ in a round bottom (RB) flask $(250 \mathrm{~mL})$ and then $\mathrm{NaH}(0.045 \mathrm{~mol})$ was added. The mixture was stirred for 0.5 hours and then ethyl 2-bromoacetate $(0.045 \mathrm{~mol})$ was added. The stirring was continued for 3-4 hours along with monitoring through TLC. Excess cold distilled water was added and the formed precipitates were filtered out, washed, and dried.

2.4. Synthesis of 2-[(6-Chloro-4-methyl-2-oxo-2H-chromen-7yl)oxy] acetohydrazide (5). The ethyl ester $4(0.04 \mathrm{~mol})$ was mixed with methanol $(35 \mathrm{~mL})$ in a RB flask $(250 \mathrm{~mL})$. The hydrated hydrazine $(0.04 \mathrm{~mol})$ was added and the mixture was set to stir for 2-3 hours. TLC was frequently developed to monitor the reaction. Solvent was evaporated to one-third and then excess of distilled water was added to precipitate the product. The precipitates were acquired through filtration and subjected to washing and drying.

2.5. Synthesis of 5-\{[(6-Chloro-4-methyl-2-oxo-2H-chromen7-yl)oxy] methyl\}-1,3,4-oxadiazol-2-thiol (6). Compound 5 $(0.035 \mathrm{~mol})$ was added to absolute ethanol $(50 \mathrm{~mL})$ in a $\mathrm{RB}$ flask $(250 \mathrm{~mL})$ followed by solid $\mathrm{KOH}(0.035 \mathrm{~mol})$. The mixture was homogenized on reflux and then cooled to room temperature and liquid $\mathrm{CS}_{2}(0.07 \mathrm{~mol})$ was added. The mixture was again set to reflux for 4-5 hours along with supervision by TLC. Solvent was evaporated to one-third and then excess cold distilled water was added. The $\mathrm{pH}$ of this homogeneous solution was adjusted to 6-7 by dilute $\mathrm{HCl}$ and aged for 0.5 hours to allow maximum precipitation. The precipitates were filtered, washed with distilled water, and dried.

2.6. General Synthesis of $\mathrm{N}$-Aralkyl/alkyl/aryl-2-bromoacetamide $(\mathbf{8} \boldsymbol{a}-\boldsymbol{o})$. Aralkyl/alkyl/aryl amines (0.005 mol; 7ao) were dispersed in distilled water $(15 \mathrm{~mL})$ in an iodine flask $(125 \mathrm{~mL})$. The $\mathrm{pH}$ was adjusted to $8-9$ by aqueous $\mathrm{Na}_{2} \mathrm{CO}_{3}$ solution $(15 \%, 4 \mathrm{~mL})$. Then 2 -bromoacetyl bromide $(0.005 \mathrm{~mol})$ was added on vigorous stirring and further set to stir for 1 hour on maintained $\mathrm{pH}$. The precipitates of title products were filtered off, washed by distilled water and dried.

2.7. General Synthesis of $\mathrm{N}$-Aralkyl/alkyl/aryl-2-[(5-\{[(6chloro-4-methyl-2-oxo-2H-chromen-7-yl)oxy]methyl $\}-1,3,4-$ oxadiazol-2-yl)sulfanyl]acetamide (9a-o). Compound $\mathbf{6}$ $(0.002 \mathrm{~mol})$ was dissolved in $\mathrm{DMF}(12 \mathrm{~mL})$ in a round bottom flask $(50 \mathrm{~mL})$ followed by $\mathrm{NaH}(0.002 \mathrm{~mol})$. The mixture was stirred for 0.5 hours and then $N$-aralkyl/alkyl/aryl2-bromoacetamide $(0.002 \mathrm{~mol} ; \mathbf{8 a}-\mathbf{o})$ were added. The stirring was continued for 4-6 hours along with monitoring through TLC. Excess cold distilled water was added and the precipitates were filtered out, washed, and dried.

2.8. Antibacterial Activity Assay. The sterilized 96-well microplates under aseptic conditions were used to evaluate the antibacterial activity by the reported method of Kaspady et al., 2009, and Yang et al., 2006, with slight modifications [21, 22]. The change in absorbance before and after the addition of sample compound was noted. The absorbance is varied with number of microbial cells which are varied with log phase microbial growth.

The clinically isolated three Gram-negative and two Gram-positive bacteria were stored on stock culture agar medium. A mixture of $200 \mu \mathrm{L}$ was prepared by $180 \mu \mathrm{L}$ fresh nutrient broth with suitable dilutions and $20 \mu \mathrm{g}$ test samples with suitable dilutions. All the dilutions were performed using specific suited solvents. Before and after incubation at $37^{\circ} \mathrm{C}$ for $16-24 \mathrm{hrs}$ with lid on the microplate, the absorbance $(0.12-0.19$ at beginning) was measured at $540 \mathrm{~nm}$. The variation in absorbance was the criteria for bacterial growth. The percent inhibition was calculated by the following formula:

$$
\text { Inhibition }(\%)=\frac{X-Y}{X} \times 100 \text {, }
$$

where $X$ is absorbance in control with bacterial culture and $Y$ is absorbance in test sample. Ciprofloxacin was taken as reference standard. Minimum inhibitory concentration (MIC) was measured with suitable dilutions (5-30 $\mu \mathrm{g} /$ well) and results were calculated using EZ-Fit Perrella Scientific Inc., Amherst, USA, software.

2.9. Statistical Analysis. The antibacterial activity results were reported as percentage of age inhibition and minimum inhibitory concentration (MIC) values after performing each experiment three times. The results were reported as mean \pm SEM after statistical analysis by Microsoft Excel 2010. 
2.10. Characterization of the Synthesized Compounds $(3-6,9 a-o)$

2.10.1. 6-Chloro-7-hydroxy-4-methyl-2H-chromen-2-one (3). Light brown amorphous solid; Yield: 78\%; M.P.: 262-264 ${ }^{\circ} \mathrm{C}$; M.F.: $\mathrm{C}_{10} \mathrm{H}_{7} \mathrm{ClO}_{3}$; M.M.: $210 \mathrm{gmol}^{-1}$; IR (KBr, $\left.v_{\max }, \mathrm{cm}^{-1}\right)$ : $3310(\mathrm{O}-\mathrm{H}), 3055$ (Ar C-H), 1732 (ester C=O), 1586 (Ar C=C), $1146(\mathrm{C}-\mathrm{O}), 703(\mathrm{C}-\mathrm{Cl}) ;{ }^{1} \mathrm{H}-\mathrm{NMR}\left(400 \mathrm{MHz}, \mathrm{CHCl}_{3}-d_{1}, \delta\right.$, ppm): 7.54 (s, $\left.1 \mathrm{H}, \mathrm{H}-5^{\prime}\right), 6.98$ (s, $\left.1 \mathrm{H}, \mathrm{H}-8^{\prime}\right), 6.17$ (s, $1 \mathrm{H}, \mathrm{H}-$ $\left.3^{\prime}\right), 2.37\left(\mathrm{~s}, 3 \mathrm{H}, \mathrm{CH}_{3}-11^{\prime}\right) ;{ }^{13} \mathrm{C}-\mathrm{NMR}\left(125 \mathrm{MHz}, \mathrm{CHCl}_{3}-d_{1}\right.$, $\delta$, ppm): $160.5\left(\mathrm{C}-2^{\prime}\right), 157.7\left(\mathrm{C}-7^{\prime}\right), 153.4\left(\mathrm{C}-4^{\prime}\right), 151.5\left(\mathrm{C}-9^{\prime}\right)$, $125.4\left(\mathrm{C}-5^{\prime}\right), 118.7\left(\mathrm{C}-6^{\prime}\right), 113.6\left(\mathrm{C}-10^{\prime}\right), 112.7\left(\mathrm{C}-3^{\prime}\right), 100.3(\mathrm{C}-$ $\left.8^{\prime}\right), 18.5\left(\mathrm{C}-11^{\prime}\right)$; EIMS $(m / z): 212(6 \%), 210[\mathrm{M}]^{\bullet+}(17 \%), 193$ (7\%), 182 (5\%), 175 (BP, 100\%), 165 (3\%), 149 (2\%), 134 (5\%).

2.10.2. Ethyl 2-[(6-Chloro-4-methyl-2-oxo-2H-chromen-7yl)oxy]acetate (4). Cream white amorphous solid; Yield: 73\%; M.P.: $184-186^{\circ}$ C; M.F.: $\mathrm{C}_{14} \mathrm{H}_{13} \mathrm{ClO}_{5}$; M.M.: 296 gmol $^{-1}$; IR (KBr, $\left.v_{\max }, \mathrm{cm}^{-1}\right): 3316(\mathrm{O}-\mathrm{H}), 3062(\mathrm{Ar} \mathrm{C}-\mathrm{H}), 1735$ (ester $\mathrm{C}=\mathrm{O}), 1594(\mathrm{Ar} \mathrm{C}=\mathrm{C}), 1159(\mathrm{C}-\mathrm{O}), 702(\mathrm{C}-\mathrm{Cl}) ;{ }^{1} \mathrm{H}-\mathrm{NMR}$ $\left(400 \mathrm{MHz}, \mathrm{CHCl}_{3}-d_{1}, \delta, \mathrm{ppm}\right): 7.59\left(\mathrm{~s}, 1 \mathrm{H}, \mathrm{H}-5^{\prime}\right), 6.71(\mathrm{~s}$, $\left.1 \mathrm{H}, \mathrm{H}-8^{\prime}\right), 6.18\left(\mathrm{~s}, 1 \mathrm{H}, \mathrm{H}-3^{\prime}\right), 4.74\left(\mathrm{~s}, 2 \mathrm{H}, \mathrm{H}-12^{\prime}\right), 4.27$ (q, $J=$ $\left.7.2 \mathrm{~Hz}, 2 \mathrm{H}, \mathrm{H}-1^{\prime \prime \prime}\right), 2.37$ (s, 3H, $\left.\mathrm{CH}_{3}-11^{\prime}\right), 1.30(\mathrm{t}, J=7.2 \mathrm{~Hz}$, $\left.3 \mathrm{H}, \mathrm{CH}_{3}-2^{\prime \prime \prime}\right)$; EIMS ( $\left.m / z\right): 298(6 \%), 296[\mathrm{M}]^{\bullet+}(17 \%), 261$ (19\%), 251 (4\%), 210 (91\%), 193 (18\%), 182 (BP, 100\%), 165 (8\%), 149 (7\%), $134(16 \%)$.

2.10.3. 2-[(6-Chloro-4-methyl-2-oxo-2H-chromen-7-yl)oxy]acetohydrazide (5). Light yellow amorphous solid; Yield: 67\%; M.P.: 190-192 ${ }^{\circ}$ C; M.F.: $\mathrm{C}_{12} \mathrm{H}_{11} \mathrm{ClN}_{2} \mathrm{O}_{4}$; M.M.: 282 gmol $^{-1}$; IR $\left(\mathrm{KBr}, v_{\max }, \mathrm{cm}^{-1}\right): 3421(\mathrm{~N}-\mathrm{H}), 3061$ (Ar C-H), 1735 (ester $\mathrm{C}=\mathrm{O}), 1671$ (amide $\mathrm{C}=\mathrm{O}), 1594$ (Ar C=C), 1159 (C-O), 703 (C-Cl); ${ }^{1} \mathrm{H}-\mathrm{NMR}\left(400 \mathrm{MHz}, \mathrm{CHCl}_{3}-d_{1}, \delta, \mathrm{ppm}\right): 8.84$ (s, $1 \mathrm{H}$, CON-H), 7.57 (s, 1H, H-5 $\left.{ }^{\prime}\right), 6.72\left(\mathrm{~s}, 1 \mathrm{H}, \mathrm{H}-8^{\prime}\right), 6.19$ (s, 1H, H$\left.3^{\prime}\right), 4.72\left(\mathrm{~s}, 2 \mathrm{H}, \mathrm{H}-12^{\prime}\right), 2.38\left(\mathrm{~s}, 3 \mathrm{H}, \mathrm{CH}_{3}-11^{\prime}\right)$; EIMS $(m / z)$ : $284(7 \%), 282[\mathrm{M}]^{\bullet+}(16 \%), 249(7 \%), 247(18 \%), 230(4 \%)$, 214 (14\%), 210 (89\%), 193 (15\%), 182 (BP, 100\%), 165 (4\%), 149 (7\%), 134 (17\%).

2.10.4. 5-\{[(6-Chloro-4-methyl-2-oxo-2H-chromen-7-yl)oxy]meth$y l$-1,3,4-oxadiazol-2-thiol (6). Light brown amorphous solid; Yield: $73 \%$; M.P.: $188-190^{\circ} \mathrm{C}$; M.F.: $\mathrm{C}_{13} \mathrm{H}_{9} \mathrm{ClN}_{2} \mathrm{O}_{4}$ S; M.M.: $324 \mathrm{gmol}^{-1}$; IR (KBr, $\left.v_{\max }, \mathrm{cm}^{-1}\right)$ : 3078 (Ar C-H), 1736 (ester $\mathrm{C}=\mathrm{O}), 1689(\mathrm{C}=\mathrm{N}), 1595(\mathrm{Ar} \mathrm{C}=\mathrm{C}), 1158(\mathrm{C}-\mathrm{O})$, $697(\mathrm{C}-\mathrm{Cl}) ;{ }^{1} \mathrm{H}-\mathrm{NMR}\left(400 \mathrm{MHz}, \mathrm{CHCl}_{3}-d_{1}, \delta, \mathrm{ppm}\right): 7.59$ (s, $\left.1 \mathrm{H}, \mathrm{H}-5^{\prime}\right), 6.75\left(\mathrm{~s}, 1 \mathrm{H}, \mathrm{H}-8^{\prime}\right), 6.21\left(\mathrm{~s}, 1 \mathrm{H}, \mathrm{H}-3^{\prime}\right), 4.74(\mathrm{~s}$, $2 \mathrm{H}, \mathrm{H}-12^{\prime}$ ), 2.39 (s, 3H, $\left.\mathrm{CH}_{3}-11^{\prime}\right)$; EIMS $(\mathrm{m} / z)$ : 326 (7\%), 324 $[\mathrm{M}]^{\bullet+}(16 \%), 289(14 \%), 251(3 \%), 249(7 \%), 230(2 \%), 214$ (13\%), 210 (86\%), 193 (13\%), 182 (BP, 100\%), 165 (7\%), 149 (4\%), $134(16 \%)$.

2.10.5. N-Cyclohexyl-2-[(5-\{[(6-chloro-4-methyl-2-oxo- $2 H$ chromen-7-yl)oxy]methyl\}-1,3,4-oxadiazol-2-yl)sulfanyl]acetamide (9a). White amorphous solid; Yield: 78\%; M.P.: 100$102^{\circ} \mathrm{C}$; M.F.: $\mathrm{C}_{21} \mathrm{H}_{22} \mathrm{ClN}_{3} \mathrm{O}_{5}$ S; M.M.: $463 \mathrm{gmol}^{-1}$; IR (KBr, $\left.v_{\max }, \mathrm{cm}^{-1}\right): 3445(\mathrm{~N}-\mathrm{H}), 3073($ Ar C-H), 1739 (ester $\mathrm{C}=\mathrm{O})$, 1676 (amide $\mathrm{C}=\mathrm{O}), 1684(\mathrm{C}=\mathrm{N}), 1596(\mathrm{Ar} \mathrm{C}=\mathrm{C}), 1159(\mathrm{C}-\mathrm{O})$,
697 (C-Cl); ${ }^{1} \mathrm{H}-\mathrm{NMR}\left(400 \mathrm{MHz}, \mathrm{CHCl}_{3}-d_{1}, \delta, \mathrm{ppm}\right): 8.71$ (s, $1 \mathrm{H}, \mathrm{CON}-\mathrm{H}), 7.57$ (s, $\left.1 \mathrm{H}, \mathrm{H}-5^{\prime}\right), 7.04\left(\mathrm{~s}, 1 \mathrm{H}, \mathrm{H}-8^{\prime}\right), 6.23$ (s, $1 \mathrm{H}, \mathrm{H}-3^{\prime}$ ), 5.33 (s, 2H, H-12' ), 4.05 (s, 2H, H-2 $\left.{ }^{\prime \prime \prime}\right), 3.76-3.73$ $\left(\mathrm{m}, 1 \mathrm{H}, \mathrm{H}-1^{\prime \prime}\right), 2.38\left(\mathrm{~s}, 3 \mathrm{H}, \mathrm{CH}_{3}-11^{\prime}\right), 1.85-1.82\left(\mathrm{~m}, 2 \mathrm{H}, \mathrm{H}_{\mathrm{eq}}{ }^{-}\right.$ $\left.2^{\prime \prime} \& \mathrm{H}_{\mathrm{eq}}-6^{\prime \prime}\right), 1.67-1.56\left(\mathrm{~m}, 4 \mathrm{H}, \mathrm{H}-3^{\prime \prime} \& \mathrm{H}-5^{\prime \prime}\right), 1.35-1.32(\mathrm{~m}$, $\left.2 \mathrm{H}, \mathrm{H}_{\mathrm{ax}}-2^{\prime \prime} \& \mathrm{H}_{\mathrm{ax}}-6^{\prime \prime}\right), 1.22-1.16\left(\mathrm{~m}, 2 \mathrm{H}, \mathrm{H}-4^{\prime \prime}\right)$; $\operatorname{EIMS}(m / z)$ : $465(9 \%), 463[\mathrm{M}]^{\cdot+}(20 \%), 428(16 \%), 251(4 \%), 249(8 \%)$, 230 (4\%), 214 (13\%), 210 (90\%), 193 (17\%), 182 (BP, 100\%), 165 (4\%), 149 (8\%), 134 (17\%), 126 (28\%), 98 (34\%).

2.10.6. N-Benzyl-2-[(5-\{[(6-chloro-4-methyl-2-oxo-2H-chromen-7-yl)oxy]methyl\}-1,3,4-oxadiazol-2-yl)sulfanyl]acetamide (9b). Light yellow amorphous solid; Yield: 84\%; M.P.: 126$128^{\circ} \mathrm{C}$; M.F.: $\mathrm{C}_{22} \mathrm{H}_{18} \mathrm{ClN}_{3} \mathrm{O}_{5}$ S; M.M.: $471 \mathrm{gmol}^{-1}$; IR ( $\mathrm{KBr}$, $\left.v_{\max }, \mathrm{cm}^{-1}\right): 3464(\mathrm{~N}-\mathrm{H}), 3053($ Ar C-H), 1736 (ester $\mathrm{C}=\mathrm{O})$, 1672 (amide $\mathrm{C}=\mathrm{O}), 1689(\mathrm{C}=\mathrm{N}), 1609$ ( $\mathrm{Ar} \mathrm{C}=\mathrm{C}), 1156(\mathrm{C}-\mathrm{O})$, 704 (C-Cl); ${ }^{1} \mathrm{H}-\mathrm{NMR}\left(400 \mathrm{MHz}, \mathrm{CHCl}_{3}-d_{1}, \delta, \mathrm{ppm}\right): 8.64$ (s, 1H, CON-H), 7.61 (s, 1H, H-5 $), 7.25-7.19\left(\mathrm{~m}, 5 \mathrm{H}, \mathrm{H}-2^{\prime \prime}\right.$ to $\left.\mathrm{H}-6^{\prime \prime}\right), 7.06\left(\mathrm{~s}, 1 \mathrm{H}, \mathrm{H}-8^{\prime}\right), 6.23$ (s, $\left.1 \mathrm{H}, \mathrm{H}-3^{\prime}\right), 5.37$ (s, 2H, $\left.\mathrm{H}-12^{\prime}\right), 4.37$ (s, 2H, H-7 $\left.7^{\prime \prime}\right), 4.06$ (s, 2H, H-2 $\left.{ }^{\prime \prime \prime}\right), 2.34$ (s, 3H, $\left.\mathrm{CH}_{3}-11^{\prime}\right)$; EIMS $(m / z): 473(8 \%), 471[\mathrm{M}]^{\bullet+}(24 \%), 436(13 \%)$, 251 (5\%), 249 (9\%), 230 (3\%), 214 (8\%), 210 (84\%), 193 (11\%), 182 (BP, 100\%), 165 (7\%), 149 (5\%), 134 (47\%), 106 (35\%).

2.10.7. N-(2-Phenylethyl)-2-[(5-\{[(6-chloro-4-methyl-2-oxo-2Hchromen-7-yl)oxy] methyl\}-1,3,4-oxadiazol-2-yl)sulfanyl]acetamide (9c). Light grey amorphous solid; Yield: 87\%; M.P.: 126-128 ${ }^{\circ}$; M.F.: $\mathrm{C}_{23} \mathrm{H}_{20} \mathrm{ClN}_{3} \mathrm{O}_{5}$ S; M.M.: 485 gmol $^{-1}$; IR $\left(\mathrm{KBr}, v_{\max }, \mathrm{cm}^{-1}\right): 3441(\mathrm{~N}-\mathrm{H}), 3065$ (Ar C-H), 1743 (ester $\mathrm{C}=\mathrm{O}), 1659$ (amide $\mathrm{C}=\mathrm{O}), 1684(\mathrm{C}=\mathrm{N}), 1602(\mathrm{Ar} \mathrm{C}=\mathrm{C}), 1174$ (C-O), 702 (C-Cl); ${ }^{1} \mathrm{H}-\mathrm{NMR}\left(400 \mathrm{MHz}, \mathrm{CHCl}_{3}-d_{1}, \delta, \mathrm{ppm}\right)$ : $8.62(\mathrm{~s}, 1 \mathrm{H}, \mathrm{CON}-\mathrm{H}), 7.61$ (s, 1H, H-5 $\left.{ }^{\prime}\right), 7.15-7.10(\mathrm{~m}, 5 \mathrm{H}, \mathrm{H}-$ $2^{\prime \prime}$ to $\left.\mathrm{H}-6^{\prime \prime}\right), 7.05\left(\mathrm{~s}, 1 \mathrm{H}, \mathrm{H}-8^{\prime}\right), 6.21\left(\mathrm{~s}, 1 \mathrm{H}, \mathrm{H}-3^{\prime}\right), 5.34(\mathrm{~s}, 2 \mathrm{H}$, $\left.\mathrm{H}-12^{\prime}\right), 4.07$ (s, 2H, H-2 $\left.2^{\prime \prime \prime}\right), 3.43\left(\mathrm{t}, J=7.6 \mathrm{~Hz}, 2 \mathrm{H}, \mathrm{H}-8^{\prime \prime}\right), 2.73$ $\left(\mathrm{t}, J=7.6 \mathrm{~Hz}, 2 \mathrm{H}, \mathrm{H}-7^{\prime \prime}\right), 2.38\left(\mathrm{~s}, 3 \mathrm{H}, \mathrm{CH}_{3}-11^{\prime}\right)$; $\operatorname{EIMS}(\mathrm{m} / z)$ : $487(6 \%), 485[\mathrm{M}]^{\bullet+}(17 \%), 450(13 \%), 251(4 \%), 249(8 \%), 230$ (1\%), 214 (9\%), 210 (88\%), 193 (15\%), 182 (BP, 100\%), 165 (7\%), 149 (8\%), 148 (31\%), 134 (9\%), 120 (34\%).

2.10.8. N-Phenyl-2-[(5-\{[(6-chloro-4-methyl-2-oxo-2H-chromen-7-yl)oxy]methyl\}-1,3,4-oxadiazol-2-yl)sulfanyl]acetamide (9d). Light grey amorphous solid; Yield: 77\%; M.P.: 94$96^{\circ}$ C; M.F.: $\mathrm{C}_{21} \mathrm{H}_{16} \mathrm{ClN}_{3} \mathrm{O}_{5}$ S; M.M.: $457 \mathrm{gmol}^{-1}$; IR (KBr, $\left.v_{\max }, \mathrm{cm}^{-1}\right): 3436(\mathrm{~N}-\mathrm{H}), 3046($ Ar C-H), 1735 (ester $\mathrm{C}=\mathrm{O})$, 1667 (amide $\mathrm{C}=\mathrm{O}), 1681(\mathrm{C}=\mathrm{N}), 1588$ ( $\mathrm{Ar} \mathrm{C}=\mathrm{C}), 1121$ (C-O), $704(\mathrm{C}-\mathrm{Cl}) ;{ }^{1} \mathrm{H}-\mathrm{NMR}\left(400 \mathrm{MHz}, \mathrm{CHCl}_{3}-d_{1}, \delta, \mathrm{ppm}\right): 8.59$ (s, $1 \mathrm{H}, \mathrm{CON}-\mathrm{H}), 7.63$ (s, $\left.1 \mathrm{H}, \mathrm{H}-5^{\prime}\right), 7.57$ (d, $J=7.6 \mathrm{~Hz}, 2 \mathrm{H}$, $\left.\mathrm{H}-2^{\prime \prime} \& \mathrm{H}-6^{\prime \prime}\right), 7.34\left(\mathrm{t}, J=7.6 \mathrm{~Hz}, 2 \mathrm{H}, \mathrm{H}-3^{\prime \prime} \& \mathrm{H}-5^{\prime \prime}\right), 7.17$ $\left(\mathrm{t}, J=7.6 \mathrm{~Hz}, 1 \mathrm{H}, \mathrm{H}-4^{\prime \prime}\right), 7.05\left(\mathrm{~s}, 1 \mathrm{H}, \mathrm{H}-8^{\prime}\right), 6.20(\mathrm{~s}, 1 \mathrm{H}$, H-3') 5.36 (s, 2H, H-12'), 4.07 (s, 2H, H-2 ${ }^{\prime \prime \prime}$ ), 2.39 (s, 3H, $\left.\mathrm{CH}_{3}-11^{\prime}\right)$; EIMS $(m / z): 459(6 \%), 457[\mathrm{M}]^{\bullet+}(21 \%), 422(18 \%)$, 251 (6\%), 249 (10\%), 230 (4\%), 214 (12\%), 210 (87\%), 193 (15\%), 182 (BP, 100\%), 165 (7\%), 149 (4\%), 134 (17\%), 120 (37\%), 92 (29\%). 
2.10.9. N-(2-Methylphenyl)-2-[(5-\{[(6-chloro-4-methyl-2-oxo2H-chromen-7-yl)oxy] methyl\}-1,3,4-oxadiazol-2-yl)sulfanyl]acetamide (9e). White amorphous solid; Yield: 69\%; M.P.: $100-102^{\circ}$ C; M.F.: $\mathrm{C}_{22} \mathrm{H}_{18} \mathrm{ClN}_{3} \mathrm{O}_{5}$ S; M.M.: 471 gmol $^{-1}$; IR $\left(\mathrm{KBr}, v_{\max }, \mathrm{cm}^{-1}\right): 3442(\mathrm{~N}-\mathrm{H}), 3069$ (Ar C-H), 1733 (ester $\mathrm{C}=\mathrm{O}$ ), 1678 (amide $\mathrm{C}=\mathrm{O}), 1691(\mathrm{C}=\mathrm{N}), 1603$ (Ar C=C), 1150 (C-O), $701(\mathrm{C}-\mathrm{Cl}) ;{ }^{1} \mathrm{H}-\mathrm{NMR}\left(400 \mathrm{MHz}, \mathrm{CHCl}_{3}-d_{1}, \delta, \mathrm{ppm}\right)$ : $8.73(\mathrm{~s}, 1 \mathrm{H}, \mathrm{CON}-\mathrm{H}), 7.72\left(\mathrm{~d}, J=8.0 \mathrm{~Hz}, 1 \mathrm{H}, \mathrm{H}-6^{\prime \prime}\right), 7.63(\mathrm{~s}$, $\left.1 \mathrm{H}, \mathrm{H}-5^{\prime}\right), 7.21\left(\mathrm{~d}, J=8.0 \mathrm{~Hz}, 1 \mathrm{H}, \mathrm{H}-3^{\prime \prime}\right), 7.15(\mathrm{t}, J=8.0 \mathrm{~Hz}, 1 \mathrm{H}$, H-5 $\left.5^{\prime \prime}\right), 7.07$ (t, $\left.J=8.0 \mathrm{~Hz}, 1 \mathrm{H}, \mathrm{H}-4^{\prime \prime}\right), 7.02\left(\mathrm{~s}, 1 \mathrm{H}, \mathrm{H}-8^{\prime}\right), 6.22$ $\left(\mathrm{s}, 1 \mathrm{H}, \mathrm{H}-3^{\prime}\right), 5.34$ (s, 2H, H-12' $), 4.06$ (s, 2H, H-2 $\left.{ }^{\prime \prime \prime}\right), 2.38$ (s, $\left.3 \mathrm{H}, \mathrm{CH}_{3}-11^{\prime}\right), 2.28\left(\mathrm{~s}, 3 \mathrm{H}, \mathrm{CH}_{3}-7^{\prime \prime}\right)$; EIMS $(\mathrm{m} / \mathrm{z})$ : $473(7 \%)$, $471[\mathrm{M}]^{\circ+}(24 \%), 436(12 \%), 251(4 \%), 249(7 \%), 230(5 \%)$, 214 (16\%), 210 (81\%), 193 (13\%), 182 (BP, 100\%), 165 (8\%), 149 (3\%), 134 (47\%), 106 (32\%).

2.10.10. N-(3-Methylphenyl)-2-[(5-\{[(6-chloro-4-methyl-2oxo-2H-chromen-7-yl)oxy]methyl\}-1,3,4-oxadiazol-2-yl)sulfanyl]acetamide (9f). Yellowish grey amorphous solid; Yield: $83 \%$; M.P.: $88-90^{\circ}$ C; M.F.: $\mathrm{C}_{22} \mathrm{H}_{18} \mathrm{ClN}_{3} \mathrm{O}_{5}$ S; M.M.: $471 \mathrm{gmol}^{-1}$; IR $\left(\mathrm{KBr}, v_{\max }, \mathrm{cm}^{-1}\right): 3432(\mathrm{~N}-\mathrm{H}), 3067(\mathrm{Ar}$ $\mathrm{C}-\mathrm{H}), 1736$ (ester $\mathrm{C}=\mathrm{O}), 1668$ (amide $\mathrm{C}=\mathrm{O}), 1689(\mathrm{C}=\mathrm{N})$, 1591 (Ar C=C), 1153 (C-O), 703 (C-Cl); ${ }^{1} \mathrm{H}-\mathrm{NMR}(400 \mathrm{MHz}$, $\left.\mathrm{CHCl}_{3}-d_{1}, \delta, \mathrm{ppm}\right): 8.82(\mathrm{~s}, 1 \mathrm{H}, \mathrm{CON}-\mathrm{H}), 7.61$ (s, $\left.1 \mathrm{H}, \mathrm{H}-5^{\prime}\right)$, $7.33\left(\mathrm{~s}, 1 \mathrm{H}, \mathrm{H}-2^{\prime \prime}\right), 7.30\left(\mathrm{~d}, J=7.6 \mathrm{~Hz}, 1 \mathrm{H}, \mathrm{H}-6^{\prime \prime}\right), 7.17(\mathrm{t}, J=$ $\left.8.0 \mathrm{~Hz}, 1 \mathrm{H}, \mathrm{H}-5^{\prime \prime}\right), 7.04\left(\mathrm{~s}, 1 \mathrm{H}, \mathrm{H}-8^{\prime}\right), 6.91(\mathrm{~d}, J=7.6 \mathrm{~Hz}, 1 \mathrm{H}$, $\mathrm{H}-4^{\prime \prime}$ ), 6.21 (s, $\left.1 \mathrm{H}, \mathrm{H}-3^{\prime}\right), 5.34$ (s, 2H, H-12' $), 3.98$ (s, 2H, $\left.\mathrm{H}-2^{\prime \prime \prime}\right), 2.37$ (s, 3H, $\left.\mathrm{CH}_{3}-11^{\prime}\right), 2.30$ (s, 3H, $\left.\mathrm{CH}_{3}-7^{\prime \prime}\right)$; EIMS $(\mathrm{m} / z): 473(5 \%), 471[\mathrm{M}]^{\circ+}(19 \%), 436(13 \%), 251(7 \%), 249$ (8\%), 230 (2\%), 214 (15\%), 210 (83\%), 193 (14\%), 182 (BP, 100\%), 165 (9\%), 149 (5\%), 134 (42\%), 106 (28\%).

2.10.11. N-(4-Methylphenyl)-2-[(5-\{[(6-chloro-4-methyl-2oxo-2H-chromen-7-yl)oxy] methyl\}-1,3,4-oxadiazol-2-yl)sulfanyl]acetamide (9g). White amorphous solid; Yield: 79\%; M.P.: $98-100^{\circ}$ C; M.F.: $\mathrm{C}_{22} \mathrm{H}_{18} \mathrm{ClN}_{3} \mathrm{O}_{5}$ S; M.M.: 471 gmol $^{-1}$; IR $\left(\mathrm{KBr}, v_{\max }, \mathrm{cm}^{-1}\right): 3435(\mathrm{~N}-\mathrm{H}), 3061$ (Ar C-H), 1734 (ester $\mathrm{C}=\mathrm{O}$ ), 1668 (amide $\mathrm{C}=\mathrm{O}), 1689(\mathrm{C}=\mathrm{N}), 1584$ (Ar $\mathrm{C}=\mathrm{C}), 1141$ (C-O), 703 (C-Cl); ${ }^{1} \mathrm{H}-\mathrm{NMR}\left(400 \mathrm{MHz}, \mathrm{CHCl}_{3}-d_{1}, \delta, \mathrm{ppm}\right)$ : 8.66 (s, $1 \mathrm{H}, \mathrm{CON}-\mathrm{H}), 7.59$ (s, $\left.1 \mathrm{H}, \mathrm{H}-5^{\prime}\right), 7.36$ (d, $J=8.4 \mathrm{~Hz}$, $\left.2 \mathrm{H}, \mathrm{H}-2^{\prime \prime} \& \mathrm{H}-6^{\prime \prime}\right), 7.17\left(\mathrm{~d}, J=8.4 \mathrm{~Hz}, 2 \mathrm{H}, \mathrm{H}-3^{\prime \prime} \& \mathrm{H}-5^{\prime \prime}\right)$, 7.05 (s, 1H, H-8 ${ }^{\prime}$ ), 6.21 (s, 1H, H-3'), 5.34 (s, 2H, H-12' $), 4.06$ (s, 2H, H-2' $\left.2^{\prime \prime \prime}\right), 2.36$ (s, 3H, $\left.\mathrm{CH}_{3}-11^{\prime}\right), 2.26$ (s, 3H, $\left.\mathrm{CH}_{3}-7^{\prime \prime}\right)$; EIMS $(\mathrm{m} / z): 473(8 \%), 471[\mathrm{M}]^{\circ+}(23 \%), 436(15 \%), 251(7 \%)$, 249 (9\%), 230 (3\%), 214 (15\%), 210 (87\%), 193 (14\%), 182 (BP, 100\%), 165 (9\%), 149 (4\%), 134 (49\%), 106 (31\%).

2.10.12. N-(2-Ethylphenyl)-2-[(5-\{[(6-chloro-4-methyl-2-oxo2H-chromen-7-yl)oxy] methyl\}-1,3,4-oxadiazol-2-yl)sulfanyl]acetamide (9h). Light brown amorphous solid; Yield: 84\%; M.P.: 92-94 ${ }^{\circ}$; M.F.: $\mathrm{C}_{23} \mathrm{H}_{20} \mathrm{ClN}_{3} \mathrm{O}_{5}$ S; M.M.: 485 gmol $^{-1}$; IR $\left(\mathrm{KBr}, v_{\max }, \mathrm{cm}^{-1}\right): 3447(\mathrm{~N}-\mathrm{H}), 3069$ (Ar C-H), 1738 (ester $\mathrm{C}=\mathrm{O}), 1677$ (amide $\mathrm{C}=\mathrm{O}), 1689(\mathrm{C}=\mathrm{N}), 1606(\mathrm{Ar} \mathrm{C}=\mathrm{C}), 1149$ (C-O), 705 (C-Cl); ${ }^{1} \mathrm{H}-\mathrm{NMR}\left(400 \mathrm{MHz}, \mathrm{CHCl}_{3}-d_{1}, \delta, \mathrm{ppm}\right)$ : $8.61(\mathrm{~s}, 1 \mathrm{H}, \mathrm{CON}-\mathrm{H}), 7.58\left(\mathrm{~s}, 1 \mathrm{H}, \mathrm{H}-5^{\prime}\right), 7.16(\mathrm{~d}, J=8.0 \mathrm{~Hz}, 1 \mathrm{H}$, $\left.\mathrm{H}-6^{\prime \prime}\right), 7.11\left(\mathrm{t}, J=8.0 \mathrm{~Hz}, 1 \mathrm{H}, \mathrm{H}-5^{\prime \prime}\right), 7.07(\mathrm{t}, J=8.0 \mathrm{~Hz}, 1 \mathrm{H}$, $\left.\mathrm{H}-4^{\prime \prime}\right), 7.04$ (s, $\left.1 \mathrm{H}, \mathrm{H}-8^{\prime}\right), 6.98$ (d, $\left.J=8.0 \mathrm{~Hz}, 1 \mathrm{H}, \mathrm{H}-3^{\prime \prime}\right), 6.21$ $\left(\mathrm{s}, 1 \mathrm{H}, \mathrm{H}-3^{\prime}\right), 5.35\left(\mathrm{~s}, 2 \mathrm{H}, \mathrm{H}-12^{\prime}\right), 4.09\left(\mathrm{~s}, 2 \mathrm{H}, \mathrm{H}-2^{\prime \prime \prime}\right), 2.46$ (q,
$\left.J=7.2 \mathrm{~Hz}, 2 \mathrm{H}, \mathrm{H}-7^{\prime \prime}\right), 2.36\left(\mathrm{~s}, 3 \mathrm{H}, \mathrm{CH}_{3}-11^{\prime}\right), 1.03(\mathrm{t}, J=7.2 \mathrm{~Hz}$, $\left.3 \mathrm{H}, \mathrm{CH}_{3}-8^{\prime \prime}\right)$; EIMS $(m / z): 487(6 \%), 485[\mathrm{M}]^{\circ+}(17 \%), 450$ (13\%), 251 (4\%), 249 (5\%), $230(2 \%), 214$ (13\%), $210(88 \%)$, 193 (14\%), 182 (BP, 100\%), 165 (6\%), 149 (7\%), 148 (33\%), 134 (16\%), $120(32 \%)$.

2.10.13. N-(4-Ethylphenyl)-2-[(5-\{[(6-chloro-4-methyl-2-oxo2H-chromen-7-yl)oxy]methyl\}-1,3,4-oxadiazol-2-yl)sulfanyl]acetamide (9i). Cream white amorphous solid; Yield: $84 \%$; M.P.: 104-106 C; M.F.: $\mathrm{C}_{23} \mathrm{H}_{20} \mathrm{ClN}_{3} \mathrm{O}_{5}$ S; M.M.: 485 gmol $^{-1}$; IR $\left(\mathrm{KBr}, v_{\max }, \mathrm{cm}^{-1}\right): 3432(\mathrm{~N}-\mathrm{H}), 3067$ (Ar C-H), 1736 (ester $\mathrm{C}=\mathrm{O}), 1671$ (amide $\mathrm{C}=\mathrm{O}), 1689(\mathrm{C}=\mathrm{N}), 1606(\mathrm{Ar} \mathrm{C}=\mathrm{C}), 1147$ (C-O), $702(\mathrm{C}-\mathrm{Cl}) ;{ }^{1} \mathrm{H}-\mathrm{NMR}\left(400 \mathrm{MHz}, \mathrm{CHCl}_{3}-d_{1}, \delta, \mathrm{ppm}\right)$ : $8.72(\mathrm{~s}, 1 \mathrm{H}, \mathrm{CON}-\mathrm{H}), 7.61\left(\mathrm{~s}, 1 \mathrm{H}, \mathrm{H}-5^{\prime}\right), 7.09(\mathrm{~d}, J=8.0 \mathrm{~Hz}$, $\left.2 \mathrm{H}, \mathrm{H}-2^{\prime \prime} \& \mathrm{H}-6^{\prime \prime}\right), 7.04\left(\mathrm{~s}, 1 \mathrm{H}, \mathrm{H}-8^{\prime}\right), 6.96(\mathrm{~d}, J=8.0 \mathrm{~Hz}$, $\left.2 \mathrm{H}, \mathrm{H}-3^{\prime \prime} \& \mathrm{H}-5^{\prime \prime}\right), 6.21$ (s, $\left.1 \mathrm{H}, \mathrm{H}-3^{\prime}\right), 5.36$ (s, 2H, H-12'), 4.08 (s, 2H, H-2 $\left.2^{\prime \prime \prime}\right), 2.54\left(\mathrm{q}, J=7.2 \mathrm{~Hz}, 2 \mathrm{H}, \mathrm{H}-7^{\prime \prime}\right), 2.36$ (s, $\left.3 \mathrm{H}, \mathrm{CH}_{3}-11^{\prime}\right), 1.13\left(\mathrm{t}, J=7.2 \mathrm{~Hz}, 3 \mathrm{H}, \mathrm{CH}_{3}-8^{\prime \prime}\right)$; EIMS $(m / z)$ : $487(8 \%), 485[\mathrm{M}]^{\bullet+}(17 \%), 450(15 \%), 251(4 \%), 249(7 \%)$, 230 (5\%), 214 (9\%), 210 (92\%), 193 (17\%), 182 (BP, 100\%), 165 (7\%), 149 (5\%), 148 (35\%), 134 (11\%), 120 (38\%).

2.10.14. N-(2-Methoxyphenyl)-2-[(5-\{[(6-chloro-4-methyl-2-oxo2H-chromen-7-yl)oxy]methyl\}-1,3,4-oxadiazol-2-yl)sulfanyl]acetamide (9j). Cream yellow amorphous solid; Yield: 78\%; M.P.: $\quad 106-108^{\circ} \mathrm{C}$; M.F.: $\mathrm{C}_{22} \mathrm{H}_{18} \mathrm{ClN}_{3} \mathrm{O}_{6}$ S; M.M.: $487 \mathrm{gmol}^{-1}$; IR (KBr, $\left.v_{\max }, \mathrm{cm}^{-1}\right): 3437(\mathrm{~N}-\mathrm{H}), 3069(\mathrm{Ar}$ $\mathrm{C}-\mathrm{H}), 1733$ (ester $\mathrm{C}=\mathrm{O}), 1683$ (amide $\mathrm{C}=\mathrm{O}), 1689(\mathrm{C}=\mathrm{N})$, 1603 (Ar C=C), $1158(\mathrm{C}-\mathrm{O}), 703(\mathrm{C}-\mathrm{Cl}) ;{ }^{1} \mathrm{H}-\mathrm{NMR}(400 \mathrm{MHz}$, $\left.\mathrm{CHCl}_{3}-d_{1}, \delta, \mathrm{ppm}\right): 8.59(\mathrm{~s}, 1 \mathrm{H}, \mathrm{CON}-\mathrm{H}), 8.22(\mathrm{~d}, J=8.0 \mathrm{~Hz}$, $\left.1 \mathrm{H}, \mathrm{H}-6^{\prime \prime}\right), 7.62$ (s, 1H, H-5'), 7.05 (s, 1H, H- $\left.8^{\prime}\right), 7.01$ (t, $J=$ $\left.7.6 \mathrm{~Hz}, 1 \mathrm{H}, \mathrm{H}-5^{\prime \prime}\right), 6.92\left(\mathrm{t}, J=7.6 \mathrm{~Hz}, 1 \mathrm{H}, \mathrm{H}-4^{\prime \prime}\right), 6.81(\mathrm{~d}, J=$ $\left.8.0 \mathrm{~Hz}, 1 \mathrm{H}, \mathrm{H}-3^{\prime \prime}\right), 6.21\left(\mathrm{~s}, 1 \mathrm{H}, \mathrm{H}-3^{\prime}\right), 5.34\left(\mathrm{~s}, 2 \mathrm{H}, \mathrm{H}-12^{\prime}\right), 4.06$ (s, 2H, H-2 $\left.2^{\prime \prime \prime}\right), 3.82\left(\mathrm{~s}, 3 \mathrm{H}, \mathrm{CH}_{3}-7^{\prime \prime}\right), 2.37$ (s, 3H, $\left.\mathrm{CH}_{3}-11^{\prime}\right)$; EIMS $(m / z): 489(5 \%), 487[\mathrm{M}]^{\circ+}(17 \%), 452(16 \%), 251(4 \%)$, 249 (6\%), 230 (2\%), 214 (13\%), 210 (86\%), 193 (12\%), 182 (BP, 100\%), 165 (4\%), 150 (31\%), 149 (8\%), 134 (18\%), 122 (39\%).

2.10.15. N-(2-Ethoxyphenyl)-2-[(5-\{[(6-chloro-4-methyl-2oxo-2H-chromen-7-yl)oxy] methyl\}-1,3,4-oxadiazol-2-yl)sulfanyl] acetamide (9k). Light grey amorphous solid; Yield: $86 \%$; M.P.: 96-98 ${ }^{\circ}$; M.F.: $\mathrm{C}_{23} \mathrm{H}_{20} \mathrm{ClN}_{3} \mathrm{O}_{6}$ S; M.M.: 501 gmol $^{-1}$; IR $\left(\mathrm{KBr}, v_{\max }, \mathrm{cm}^{-1}\right): 3453(\mathrm{~N}-\mathrm{H}), 3056(\mathrm{Ar} \mathrm{C}-\mathrm{H}), 1736$ (ester $\mathrm{C}=\mathrm{O}), 1667$ (amide $\mathrm{C}=\mathrm{O}), 1681(\mathrm{C}=\mathrm{N}), 1606(\mathrm{Ar} \mathrm{C}=\mathrm{C}), 1155$ (C-O), 702 (C-Cl); ${ }^{1} \mathrm{H}-\mathrm{NMR}\left(400 \mathrm{MHz}, \mathrm{CHCl}_{3}-d_{1}, \delta, \mathrm{ppm}\right)$ : 8.66 (s, $1 \mathrm{H}, \mathrm{CON}-\mathrm{H}), 7.59$ (s, $\left.1 \mathrm{H}, \mathrm{H}-5^{\prime}\right), 7.43(\mathrm{~d}, J=8.4 \mathrm{~Hz}, 1 \mathrm{H}$, H-6 $\left.{ }^{\prime \prime}\right), 7.17\left(\mathrm{t}, J=8.4 \mathrm{~Hz}, 1 \mathrm{H}, \mathrm{H}-4^{\prime \prime}\right), 7.03\left(\mathrm{~s}, 1 \mathrm{H}, \mathrm{H}-8^{\prime}\right), 6.84(\mathrm{t}$, $\left.J=8.4 \mathrm{~Hz}, 1 \mathrm{H}, \mathrm{H}-5^{\prime \prime}\right), 6.75\left(\mathrm{~d}, J=8.0 \mathrm{~Hz}, 1 \mathrm{H}, \mathrm{H}-3^{\prime \prime}\right), 6.20(\mathrm{~s}$, $\left.1 \mathrm{H}, \mathrm{H}-3^{\prime}\right), 5.35$ (s, 2H, H-12' $), 4.04$ (s, 2H, H-2 $\left.2^{\prime \prime \prime}\right), 3.74$ (q, J = $\left.7.2 \mathrm{~Hz}, 2 \mathrm{H}, \mathrm{H}-7^{\prime \prime}\right), 2.37\left(\mathrm{~s}, 3 \mathrm{H}, \mathrm{CH}_{3}-11^{\prime}\right), 1.11(\mathrm{t}, J=7.2 \mathrm{~Hz}, 3 \mathrm{H}$, $\left.\mathrm{CH}_{3}-8^{\prime \prime}\right)$; EIMS $(\mathrm{m} / z): 503(8 \%), 501[\mathrm{M}]^{\bullet+}(22 \%), 466(11 \%)$, 251 (4\%), 249 (7\%), 230 (2\%), 214 (14\%), 210 (85\%), 193 (18\%), 182 (BP, 100\%), 165 (8\%), 164 (30\%), 149 (9\%), 136 (28\%), 134 (17\%).

2.10.16. N-(4-Ethoxyphenyl)-2-[(5-\{[(6-chloro-4-methyl-2-oxo2H-chromen-7-yl)oxy]methyl\}-1,3,4-oxadiazol-2-yl)sulfanyl]acetamide (9l). Reddish purple amorphous solid; Yield: 74\%; 
M.P.: $108-110^{\circ}$ C; M.F.: $\mathrm{C}_{23} \mathrm{H}_{20} \mathrm{ClN}_{3} \mathrm{O}_{6}$ S; M.M.: $501 \mathrm{gmol}^{-1}$; IR $\left(\mathrm{KBr}, v_{\max }, \mathrm{cm}^{-1}\right): 3443(\mathrm{~N}-\mathrm{H}), 3078(\mathrm{Ar} \mathrm{C}-\mathrm{H}), 1734$ (ester $\mathrm{C}=\mathrm{O}), 1679$ (amide $\mathrm{C}=\mathrm{O}), 1688(\mathrm{C}=\mathrm{N}), 1597(\mathrm{Ar} \mathrm{C}=\mathrm{C}), 1155$ (C-O), 705 (C-Cl); ${ }^{1} \mathrm{H}-\mathrm{NMR}\left(400 \mathrm{MHz}, \mathrm{CHCl}_{3}-d_{1}, \delta\right.$, ppm): $8.82(\mathrm{~s}, 1 \mathrm{H}, \mathrm{CON}-\mathrm{H}), 7.59\left(\mathrm{~s}, 1 \mathrm{H}, \mathrm{H}-5^{\prime}\right), 7.39(\mathrm{~d}, J=8.0 \mathrm{~Hz}$, $\left.2 \mathrm{H}, \mathrm{H}-2^{\prime \prime} \& \mathrm{H}-6^{\prime \prime}\right), 7.03\left(\mathrm{~s}, 1 \mathrm{H}, \mathrm{H}-8^{\prime}\right), 6.81(\mathrm{~d}, J=8.4 \mathrm{~Hz}, 2 \mathrm{H}$, $\left.\mathrm{H}-3^{\prime \prime} \& \mathrm{H}-5^{\prime \prime}\right), 6.20\left(\mathrm{~s}, 1 \mathrm{H}, \mathrm{H}-3^{\prime}\right), 5.34\left(\mathrm{~s}, 2 \mathrm{H}, \mathrm{H}-12^{\prime}\right), 3.98(\mathrm{~s}$, $\left.2 \mathrm{H}, \mathrm{H}-2^{\prime \prime \prime}\right), 3.97\left(\mathrm{q}, J=6.8 \mathrm{~Hz}, 2 \mathrm{H}, \mathrm{H}-7^{\prime \prime}\right), 2.37$ (s, $3 \mathrm{H}, \mathrm{CH}_{3}-$ $\left.11^{\prime}\right), 0.86\left(\mathrm{t}, J=6.8 \mathrm{~Hz}, 3 \mathrm{H}, \mathrm{CH}_{3}-8^{\prime \prime}\right)$; EIMS $(\mathrm{m} / z)$ : $503(9 \%)$, $501\left[\mathrm{M}^{++}(21 \%), 466(13 \%), 251(6 \%), 249(8 \%), 230(3 \%)\right.$, 214 (15\%), 210 (81\%), 193 (19\%), 182 (BP, 100\%), 165 (9\%), 164 (32\%), 149 (6\%), 136 (25\%), 134 (19\%).

2.10.17. N-(2-Methoxycarbonylphenyl)-2-[(5-\{[(6-chloro-4-methyl-2-oxo-2H-chromen-7-yl)oxy]methyl\}-1,3,4-oxadiazol-2yl)sulfanyl]acetamide $(\mathbf{9 m})$. Yellow amorphous solid; Yield: $75 \%$; M.P.: $122-124^{\circ} \mathrm{C}$; M.F.: $\mathrm{C}_{23} \mathrm{H}_{18} \mathrm{ClN}_{3} \mathrm{O}_{7}$ S; M.M.: $515 \mathrm{gmol}^{-1}$; IR $\left(\mathrm{KBr}, v_{\max }, \mathrm{cm}^{-1}\right): 3429(\mathrm{~N}-\mathrm{H}), 3050(\mathrm{Ar}$ $\mathrm{C}-\mathrm{H}), 1731$ (ester $\mathrm{C}=\mathrm{O}), 1671$ (amide $\mathrm{C}=\mathrm{O}), 1683(\mathrm{C}=\mathrm{N})$, 1601 (Ar C=C), 1159 (C-O), $703(\mathrm{C}-\mathrm{Cl}) ;{ }^{1} \mathrm{H}-\mathrm{NMR}(400 \mathrm{MHz}$, $\left.\mathrm{CHCl}_{3}-d_{1}, \delta, \mathrm{ppm}\right): 8.81(\mathrm{~s}, 1 \mathrm{H}, \mathrm{CON}-\mathrm{H}), 8.68(\mathrm{~d}, J=8.4 \mathrm{~Hz}$, $\left.1 \mathrm{H}, \mathrm{H}-6^{\prime \prime}\right), 8.10\left(\mathrm{~d}, J=7.6 \mathrm{~Hz}, 1 \mathrm{H}, \mathrm{H}-3^{\prime \prime}\right), 7.62\left(\mathrm{~s}, 1 \mathrm{H}, \mathrm{H}-5^{\prime}\right)$, $7.51\left(\mathrm{t}, J=7.6 \mathrm{~Hz}, 1 \mathrm{H}, \mathrm{H}-5^{\prime \prime}\right), 7.18\left(\mathrm{t}, J=7.6 \mathrm{~Hz}, 1 \mathrm{H}, \mathrm{H}-4^{\prime \prime}\right)$, 7.05 (s, 1H, H-8 $\left.{ }^{\prime}\right), 6.21\left(\mathrm{~s}, 1 \mathrm{H}, \mathrm{H}-3^{\prime}\right), 5.36\left(\mathrm{~s}, 2 \mathrm{H}, \mathrm{H}-12^{\prime}\right), 4.07$ (s, $\left.2 \mathrm{H}, \mathrm{H}-2^{\prime \prime \prime}\right), 3.81\left(\mathrm{~s}, 3 \mathrm{H}, \mathrm{CH}_{3}-8^{\prime \prime}\right), 2.37\left(\mathrm{~s}, 3 \mathrm{H}, \mathrm{CH}_{3}-11^{\prime}\right)$; EIMS $(\mathrm{m} / \mathrm{z}): 517(9 \%), 515[\mathrm{M}]^{\circ+}(19 \%), 480(13 \%), 251(6 \%)$, 249 (9\%), 230 (2\%), 214 (14\%), 210 (92\%), 193 (17\%), 182 (BP, 100\%), 178 (37\%), 165 (6\%), 150 (33\%), 149 (7\%), 134 (15\%).

2.10.18. N-(4-Bromophenyl)-2-[(5-\{[(6-chloro-4-methyl-2-oxo2H-chromen-7-yl)oxy] methyl\}-1,3,4-oxadiazol-2-yl)sulfanyl]acetamide $(\mathbf{9 n})$. Light grey amorphous solid; Yield: 73\%; M.P.: $102-104^{\circ}$ C; M.F.: $\mathrm{C}_{21} \mathrm{H}_{15}$ BrClN $_{3} \mathrm{O}_{5}$ S; M.M.: 535 gmol $^{-1}$; $\operatorname{IR}\left(\mathrm{KBr}, v_{\max }, \mathrm{cm}^{-1}\right): 3422(\mathrm{~N}-\mathrm{H}), 3056$ (Ar C-H), 1734 (ester $\mathrm{C}=\mathrm{O}$ ), 1662 (amide $\mathrm{C}=\mathrm{O}), 1684(\mathrm{C}=\mathrm{N}), 1593(\mathrm{Ar} \mathrm{C}=\mathrm{C}), 1143$ (C-O), 702 (C-Cl), 639 (C-Br); ${ }^{1} \mathrm{H}-\mathrm{NMR}\left(400 \mathrm{MHz}, \mathrm{CHCl}_{3}\right.$ $\left.d_{1}, \delta, \mathrm{ppm}\right): 8.28(\mathrm{~s}, 1 \mathrm{H}, \mathrm{CON}-\mathrm{H}), 7.62\left(\mathrm{~s}, 1 \mathrm{H}, \mathrm{H}-5^{\prime}\right), 7.47(\mathrm{~d}, J=$ $\left.8.4 \mathrm{~Hz}, 2 \mathrm{H}, \mathrm{H}-2^{\prime \prime} \& \mathrm{H}-6^{\prime \prime}\right), 7.41\left(\mathrm{~d}, J=8.4 \mathrm{~Hz}, 2 \mathrm{H}, \mathrm{H}-3^{\prime \prime} \& \mathrm{H}-\right.$ $\left.5^{\prime \prime}\right), 7.05\left(\mathrm{~s}, 1 \mathrm{H}, \mathrm{H}-8^{\prime}\right), 6.22\left(\mathrm{~s}, 1 \mathrm{H}, \mathrm{H}-3^{\prime}\right), 5.34\left(\mathrm{~s}, 2 \mathrm{H}, \mathrm{H}-12^{\prime}\right)$, $4.08\left(\mathrm{~s}, 2 \mathrm{H}, \mathrm{H}-2^{\prime \prime \prime}\right), 2.36\left(\mathrm{~s}, 3 \mathrm{H}, \mathrm{CH}_{3}-11^{\prime}\right)$; EIMS $(\mathrm{m} / z): 539$ (7\%), $537(18 \%), 535[\mathrm{M}]^{\circ+}(20 \%), 500(14 \%), 251(6 \%), 249$ (8\%), 230 (4\%), 214 (13\%), 210 (92\%), 198 (33\%), $193(13 \%)$, 182 (BP, 100\%), 170 (31\%), 165 (6\%), 149 (8\%), 134 (11\%).

2.10.19. $N$-(4-Nitrophenyl) $-2-[(5-\{[(6-$ chloro-4-methyl-2-oxo2H-chromen-7-yl)oxy]methyl\}-1,3,4-oxadiazol-2-yl)sulfanyl]acetamide (9o). Light yellow amorphous solid; Yield: 81\%; M.P.: $\quad 108-110^{\circ} \mathrm{C}$; M.F.: $\mathrm{C}_{21} \mathrm{H}_{15} \mathrm{ClN}_{4} \mathrm{O}_{7} \mathrm{~S}$; M.M.: $502 \mathrm{gmol}^{-1}$; IR $\left(\mathrm{KBr}, v_{\max }, \mathrm{cm}^{-1}\right): 3438(\mathrm{~N}-\mathrm{H}), 3053(\mathrm{Ar}$ $\mathrm{C}-\mathrm{H}), 1735$ (ester $\mathrm{C}=\mathrm{O}$ ), 1678 (amide $\mathrm{C}=\mathrm{O}), 1688(\mathrm{C}=\mathrm{N})$, 1606 ( $\mathrm{Ar} \mathrm{C}=\mathrm{C}), 1163(\mathrm{C}-\mathrm{O}), 703(\mathrm{C}-\mathrm{Cl}) ;{ }^{1} \mathrm{H}-\mathrm{NMR}(400 \mathrm{MHz}$, $\left.\mathrm{CHCl}_{3}-d_{1}, \delta, \mathrm{ppm}\right): 9.34(\mathrm{~s}, 1 \mathrm{H}, \mathrm{CON}-\mathrm{H}), 8.41(\mathrm{~d}, J=8.0 \mathrm{~Hz}$, $\left.2 \mathrm{H}, \mathrm{H}-3^{\prime \prime} \& \mathrm{H}-5^{\prime \prime}\right), 8.06\left(\mathrm{~d}, J=8.0 \mathrm{~Hz}, 2 \mathrm{H}, \mathrm{H}-2^{\prime \prime} \& \mathrm{H}-6^{\prime \prime}\right)$, $7.62\left(\mathrm{~s}, 1 \mathrm{H}, \mathrm{H}-5^{\prime}\right), 7.05\left(\mathrm{~s}, 1 \mathrm{H}, \mathrm{H}-8^{\prime}\right), 6.22\left(\mathrm{~s}, 1 \mathrm{H}, \mathrm{H}-3^{\prime}\right), 5.34$ (s, $\left.2 \mathrm{H}, \mathrm{H}-12^{\prime}\right), 4.07\left(\mathrm{~s}, 2 \mathrm{H}, \mathrm{H}-2^{\prime \prime \prime}\right), 2.36\left(\mathrm{~s}, 3 \mathrm{H}, \mathrm{CH}_{3}-11^{\prime}\right)$; EIMS ( $m / z)$ : $504(5 \%), 502[\mathrm{M}]^{*+}$ (17\%), 467 (18\%), $251(8 \%)$, 249 (7\%), 230 (5\%), 214 (11\%), 210 (91\%), 193 (18\%), 182 (BP, 100\%), 165 (42\%), 149 (8\%), 137 (32\%), 134 (17\%).

\section{Results and Discussion}

The different $\mathrm{N}$-aralkyl/alkyl/aryl acetamides incorporating coumarin and 1,3,4-oxadiazole rings, $\mathbf{9 a - o}$, were synthesized by the multistep protocol given in Scheme 1. The synthesis was aimed at combining multiple functionalities in a single molecule so these may be able to demonstrate more efficient biological activity. The antibacterial behavior of these molecules was tested against Gram-bacteria including positive and negative strains. The three negative and two positive bacterial strains have been reported to be the cause of various diseases [23-27].

3.1. Chemistry. First 4-chlororesorcinol (1) was treated with ethyl acetoacetate (2) in a strong acidic medium to yield 6-chloro-7-hydroxy-4-methyl-2H-chromen-2-one (3) which was separated through filtration. Compound 3 was further $\mathrm{O}$-substituted by ethyl 2-bromoacetate in a polar aprotic solvent with the aid of a weak base. This substituted ester, 4, was converted to corresponding carbohydrazide, 5, after a simple nucleophilic substitution reaction by hydrazine in methanol. The carbohydrazide was cyclized to 5 -substituted1,3,4-oxadiazol-2-thiol by $\mathrm{CS}_{2}$ in an alcoholic $\mathrm{KOH}$ medium on reflux. A list of electrophiles, 8a-o, was synthesized from different aralkyl/alkyl/aryl amines, $7 \mathbf{a}-\mathbf{o}$, on simple stirring with 2-bromoacetyl bromide in aqueous $\mathrm{Na}_{2} \mathrm{CO}_{3}$ medium. The final compounds were geared up by stirring 6 with $8 \mathbf{a}-\mathbf{o}$ again in DMF in the presence of $\mathrm{NaH}$ and separated through filtration after addition of excess distilled water.

All the compounds were structurally corroborated through IR, ${ }^{1} \mathrm{H}-\mathrm{NMR}$, and EIMS spectral data. Compound 3 is also aided by ${ }^{13} \mathrm{C}-\mathrm{NMR}$ data. One molecule description is given for $\mathbf{9 h}$. Its molecular formula was nominated as $\mathrm{C}_{23} \mathrm{H}_{20} \mathrm{ClN}_{3} \mathrm{O}_{5} \mathrm{~S}$, elucidated with the aid of molecular ion peak in EIMS spectrum and integration of protons in ${ }^{1} \mathrm{H}$ NMR spectrum. The suggested fragmentation pattern of this molecule is also sketched in Figure 1. The specific absorption bands for different functionalities in the molecule appeared in IR spectrum at $v_{\max }\left(\mathrm{cm}^{-1}\right) 3447(\mathrm{~N}-\mathrm{H}), 3069(\mathrm{Ar} \mathrm{C}-\mathrm{H})$, 1738 (ester $\mathrm{C}=\mathrm{O}), 1677$ (amide $\mathrm{C}=\mathrm{O}), 1689(\mathrm{C}=\mathrm{N}), 1606(\mathrm{Ar}$ $\mathrm{C}=\mathrm{C}), 1149(\mathrm{C}-\mathrm{O})$, and $705(\mathrm{C}-\mathrm{Cl})$. In aromatic region of ${ }^{1} \mathrm{H}-\mathrm{NMR}$ spectrum, the three singlets with single proton integration resonating at $\delta 7.58\left(\mathrm{H}-5^{\prime}\right), 7.04\left(\mathrm{H}-8^{\prime}\right)$, and 6.21 $\left(\mathrm{H}-3^{\prime}\right)$ were allocated for the chlorinated coumarin part of the molecule. The other four signals in the same region with single proton integration resonated at $\delta 7.16(\mathrm{~d}, J=$ $\left.8.0 \mathrm{~Hz}, \mathrm{H}-6^{\prime \prime}\right), 7.11\left(\mathrm{t}, J=8.0 \mathrm{~Hz}, \mathrm{H}-5^{\prime \prime}\right), 7.07(\mathrm{t}, J=8.0 \mathrm{~Hz}$, $\left.\mathrm{H}-4^{\prime \prime}\right)$, and $6.98\left(\mathrm{~d}, J=8.0 \mathrm{~Hz}, \mathrm{H}-3^{\prime \prime}\right)$ for 2-ethylphenyl part of the molecule. The ethyl group was confirmed through a quartet and a triplet, both with coupling constant of $7.2 \mathrm{~Hz}$, appearing at $\delta 2.46\left(2 \mathrm{H}, \mathrm{H}-7^{\prime \prime}\right)$ and $1.03\left(3 \mathrm{H}, \mathrm{CH}_{3}-8^{\prime \prime}\right)$, respectively, in the aliphatic region. The protons of two methylene groups linked to oxygen and sulfur appeared as two singlets at $\delta 5.35\left(\mathrm{H}-12^{\prime}\right)$ and $4.09\left(\mathrm{H}-2^{\prime \prime \prime}\right)$, respectively. The three protons of methyl group attached to coumarin nucleus resonated as singlet at $\delta 2.36\left(\mathrm{CH}_{3}-11^{\prime}\right)$. The whole discussion confirmed $\mathbf{9 h}$ as $\mathrm{N}$-(2-Ethylphenyl)-2-[(5-\{[(6chloro-4-methyl-2-oxo-2H-chromen-7-yl)oxy] methyl\}-1,3, 4-oxadiazol-2-yl)sulfanyl]acetamide. 
<smiles>CCOC(=O)CC(C)=O</smiles>

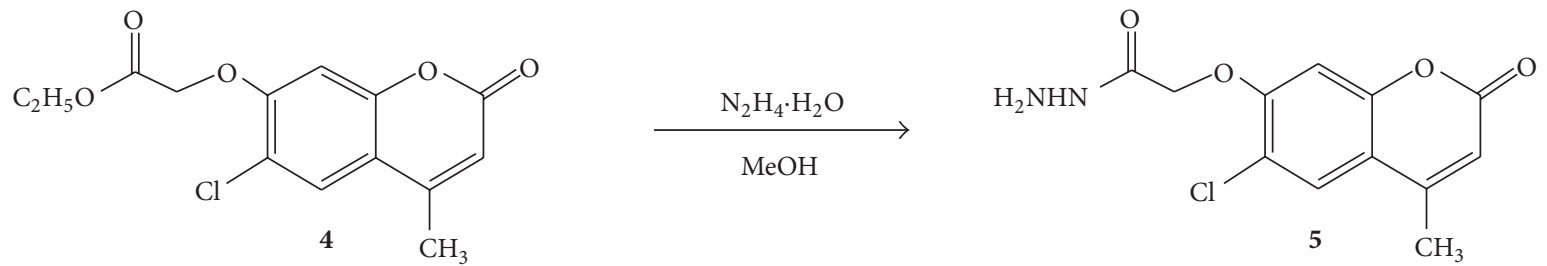

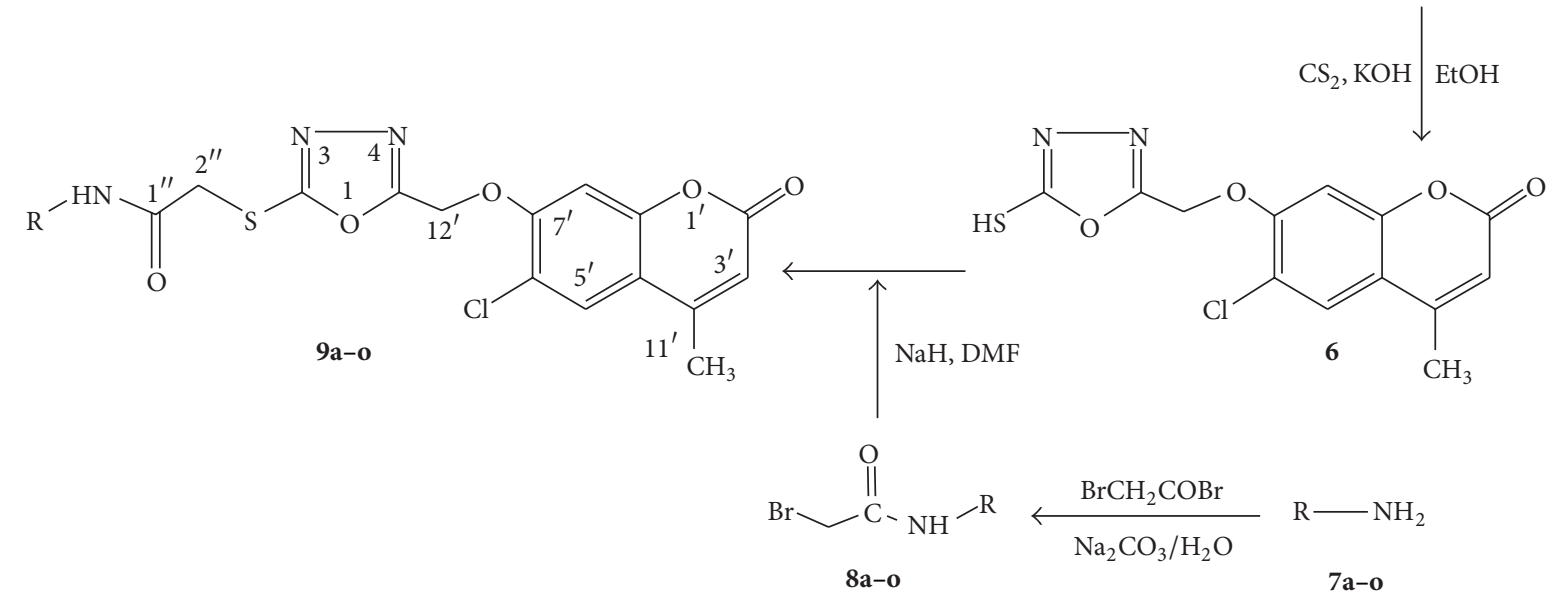

Comp.

Scheme 1: Synthesis of $N$-aralkyl/alkyl/aryl-2-[(5-\{[(6-chloro-4-methyl-2-oxo-2H-chromen-7-yl)oxy]methyl $\}$-1,3,4-oxadiazol-2-yl)sulfanyl]acetamide $(\mathbf{9} \mathbf{a}-\mathbf{o})$. 


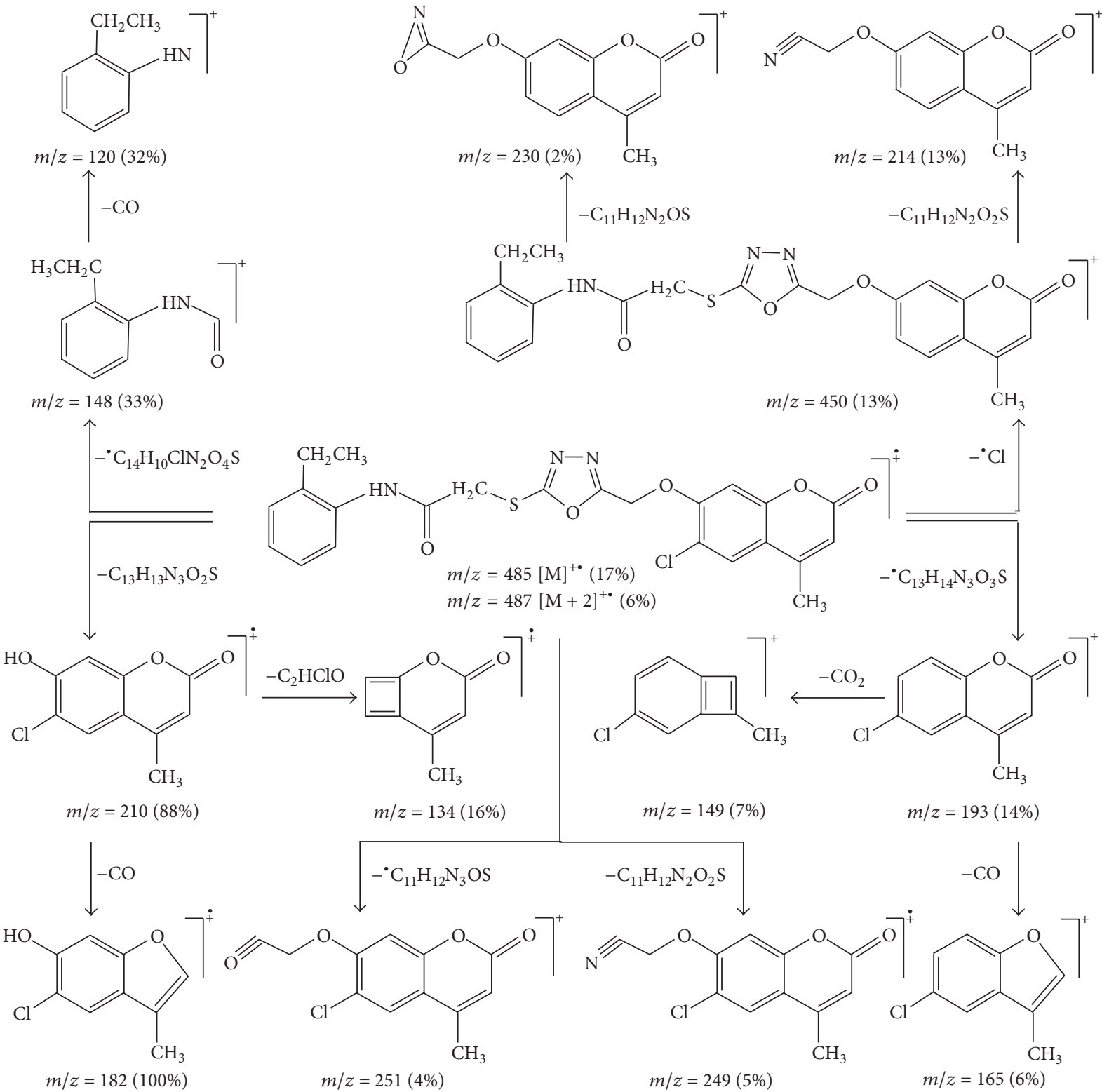

Figure 1: Mass fragmentation pattern of $N$-(2-ethylphenyl)-2-[(5-\{[(6-chloro-4-methyl-2-oxo-2H-chromen-7-yl)oxy]methyl\}-1,3,4-oxadiazol-2-yl)sulfanyl]acetamide (9h).

3.2. Antibacterial Activity (In Vitro). The results of antibacterial activity evaluation against Gram-bacteria were presented as percentage of age inhibition and MIC values in Tables 1 and 2. The ciprofloxacin was used as reference standard. All the compounds demonstrated moderate to excellent activity except a few. The Gram-negative strain, S. typhi, was not inhibited by $\mathbf{9 b}$ and $\mathbf{9 d}$. It was the most efficiently inhibited by $9 \mathrm{e}, \mathbf{9 f}, \mathbf{9 h}, \mathbf{9 j}, \mathbf{9 k}$, and $\mathbf{9 m}$. The best activity against it was observed for $9 \mathbf{j}$ with MIC value of $8.87 \pm 1.20 \mu \mathrm{g} / \mathrm{mL}$ and $9 \mathrm{k}$ as $8.84 \pm 2.00 \mu \mathrm{g} / \mathrm{mL}$ with respect to that of reference as $8.00 \pm 2.54 \mu \mathrm{g} / \mathrm{mL}$. Only 90 was inactive against $E$. coli and remaining moderate to good with the efficient ones as 9e, 9f, 9j, 9k, and $\mathbf{9 m}$. The most active one was 9 f with MIC of $10.15 \pm 4.12$ relative to $7.96 \pm 1.14 \mu \mathrm{g} / \mathrm{mL}$. Against
P. aeruginosa, $\mathbf{9 b}$ and $\mathbf{9 o}$ were inactive and remaining weakly moderate. The efficient ones against this strain were 9a, 9c, 9e, 9f, 9h, 9j, and 9m. It was most actively inhibited by $9 \mathrm{e}$ presenting MIC of $10.22 \pm 3.33 \mu \mathrm{g} / \mathrm{mL}$ as compared to $8.05 \pm 1.60 \mu \mathrm{g} / \mathrm{mL}$. B. subtilis was moderately inhibited by all the molecules and efficiently by 9 n with MIC of $11.95 \pm 4.71 \mu \mathrm{g} / \mathrm{mL}$ in comparison with $8.32 \pm 1.00 \mu \mathrm{g} / \mathrm{mL}$. The compounds against $S$. aureus were moderate to excellent inhibitors including $\mathbf{9 c}, \mathbf{9 e}, \mathbf{9 j}, \mathbf{9 k}$, and $\mathbf{9 1}$. The molecule $\mathbf{9 e}$ was the most efficient with MIC of $8.90 \pm 2.08 \mu \mathrm{g} / \mathrm{mL}$ relative to $7.43 \pm 0.45 \mu \mathrm{g} / \mathrm{mL}$. The three compounds $\mathbf{9 d}$, 9i, and $\mathbf{9 n}$ were inactive at all against this strain. Overall the Gramnegative strains were efficiently inhibited by the synthesized molecules relative to Gram-positive ones. The best activity 
TABLE 1: The $\%$ age inhibition for antibacterial activity.

\begin{tabular}{|c|c|c|c|c|c|}
\hline \multirow{3}{*}{ Compound } & \multicolumn{5}{|c|}{ Percentage inhibition (\%) } \\
\hline & \multicolumn{3}{|c|}{ Gram-negative bacteria } & \multicolumn{2}{|c|}{ Gram-positive bacteria } \\
\hline & S. typhi & E. coli & P. aeruginosa & B. subtilis & S. aureus \\
\hline 9a & $59.72 \pm 0.17$ & $58.71 \pm 0.89$ & $63.30 \pm 1.65$ & $51.35 \pm 1.05$ & $51.68 \pm 0.95$ \\
\hline $9 \mathbf{b}$ & $47.06 \pm 3.50$ & $69.65 \pm 1.14$ & $40.52 \pm 5.00$ & $75.60 \pm 3.10$ & $57.32 \pm 0.79$ \\
\hline $9 \mathrm{c}$ & $51.61 \pm 0.28$ & $66.63 \pm 0.20$ & $56.03 \pm 0.67$ & $66.55 \pm 2.25$ & $61.11 \pm 4.47$ \\
\hline 9d & $46.89 \pm 3.33$ & $50.05 \pm 4.50$ & $51.91 \pm 1.19$ & $54.35 \pm 3.05$ & $43.32 \pm 0.05$ \\
\hline $9 e$ & $74.22 \pm 0.44$ & $74.26 \pm 0.40$ & $71.80 \pm 0.26$ & $72.00 \pm 2.50$ & $67.89 \pm 0.32$ \\
\hline 9f & $73.00 \pm 0.44$ & $75.15 \pm 2.28$ & $71.91 \pm 1.39$ & $69.95 \pm 2.95$ & $64.32 \pm 0.21$ \\
\hline $9 g$ & $58.00 \pm 1.44$ & $55.54 \pm 4.85$ & $50.57 \pm 4.90$ & $56.35 \pm 2.85$ & $50.68 \pm 1.63$ \\
\hline $9 \mathrm{~h}$ & $67.56 \pm 2.22$ & $70.25 \pm 2.03$ & $68.45 \pm 0.82$ & $64.00 \pm 0.60$ & $62.21 \pm 3.05$ \\
\hline $9 \mathbf{i}$ & $51.28 \pm 2.50$ & $50.84 \pm 4.60$ & $50.05 \pm 3.66$ & $56.55 \pm 0.85$ & $47.32 \pm 0.58$ \\
\hline $9 j$ & $73.11 \pm 0.89$ & $73.22 \pm 0.15$ & $67.42 \pm 0.41$ & $64.95 \pm 1.65$ & $62.58 \pm 0.89$ \\
\hline $9 k$ & $71.89 \pm 0.44$ & $73.91 \pm 0.25$ & $67.89 \pm 2.63$ & $64.55 \pm 0.75$ & $57.32 \pm 1.95$ \\
\hline 91 & $67.56 \pm 4.94$ & $59.50 \pm 2.87$ & $53.51 \pm 4.12$ & $53.00 \pm 0.80$ & $69.21 \pm 0.89$ \\
\hline $9 \mathrm{~m}$ & $69.89 \pm 0.89$ & $71.29 \pm 0.05$ & $66.29 \pm 1.34$ & $66.35 \pm 2.25$ & $50.32 \pm 2.95$ \\
\hline $9 n$ & $60.50 \pm 0.28$ & $60.84 \pm 0.54$ & $56.80 \pm 2.37$ & $58.75 \pm 0.05$ & $43.63 \pm 2.26$ \\
\hline 90 & $50.17 \pm 1.83$ & $44.31 \pm 3.71$ & $44.85 \pm 1.13$ & $45.35 \pm 3.55$ & $53.00 \pm 2.89$ \\
\hline Ciprofloxacin & $92.43 \pm 1.07$ & $90.67 \pm 0.65$ & $90.33 \pm 0.22$ & $91.99 \pm 2.00$ & $91.00 \pm 1.65$ \\
\hline
\end{tabular}

TABLE 2: The MIC values for antibacterial activity.

\begin{tabular}{|c|c|c|c|c|c|}
\hline \multirow{3}{*}{ Compound } & \multicolumn{5}{|c|}{$\operatorname{MIC}(\mu \mathrm{g} / \mathrm{mL})$} \\
\hline & \multicolumn{3}{|c|}{ Gram-negative bacteria } & \multicolumn{2}{|c|}{ Gram-positive bacteria } \\
\hline & S. typhi & E. coli & P. aeruginosa & B. subtilis & S. aureus \\
\hline 9a & $12.59 \pm 4.60$ & $12.98 \pm 1.56$ & $11.30 \pm 2.25$ & $19.58 \pm 1.08$ & $17.86 \pm 0.65$ \\
\hline $9 b$ & - & $13.41 \pm 5.00$ & - & $14.47 \pm 5.00$ & $13.35 \pm 2.25$ \\
\hline $9 c$ & $14.46 \pm 1.10$ & $11.07 \pm 1.94$ & $11.42 \pm 3.17$ & $13.19 \pm 1.83$ & $10.91 \pm 2.87$ \\
\hline 9d & - & $19.93 \pm 1.81$ & $15.40 \pm 5.00$ & $16.51 \pm 1.98$ & - \\
\hline $9 e$ & $10.61 \pm 5.00$ & $10.39 \pm 1.62$ & $10.22 \pm 3.33$ & $12.86 \pm 3.85$ & $8.90 \pm 2.08$ \\
\hline $9 f$ & $9.50 \pm 1.30$ & $10.15 \pm 4.12$ & $10.43 \pm 4.65$ & $15.94 \pm 3.06$ & $13.18 \pm 2.17$ \\
\hline $9 g$ & $17.19 \pm 3.70$ & $14.86 \pm 5.00$ & $19.50 \pm 2.67$ & $12.12 \pm 4.00$ & $19.53 \pm 1.65$ \\
\hline $9 \mathrm{~h}$ & $9.16 \pm 2.20$ & $11.28 \pm 1.06$ & $11.52 \pm 3.42$ & $15.32 \pm 5.00$ & $12.95 \pm 3.15$ \\
\hline $9 \mathrm{i}$ & $19.07 \pm 0.40$ & $14.24 \pm 1.25$ & $19.97 \pm 3.50$ & $14.87 \pm 2.71$ & - \\
\hline $9 j$ & $8.87 \pm 1.20$ & $10.41 \pm 1.44$ & $10.54 \pm 0.80$ & $14.58 \pm 1.87$ & $11.14 \pm 2.22$ \\
\hline 9k & $8.84 \pm 2.00$ & $10.94 \pm 1.25$ & $12.00 \pm 1.33$ & $12.96 \pm 1.82$ & $12.14 \pm 1.48$ \\
\hline 91 & $12.75 \pm 4.50$ & $12.07 \pm 5.00$ & $15.61 \pm 5.00$ & $15.75 \pm 3.35$ & $11.35 \pm 2.00$ \\
\hline $9 \mathrm{~m}$ & $9.01 \pm 1.40$ & $10.86 \pm 1.19$ & $11.04 \pm 4.17$ & $14.18 \pm 4.35$ & $18.62 \pm 1.00$ \\
\hline $9 n$ & $17.64 \pm 5.00$ & $15.97 \pm 5.00$ & $17.56 \pm 2.00$ & $11.95 \pm 4.71$ & - \\
\hline 90 & $19.92 \pm 3.21$ & - & - & - & $18.32 \pm 1.11$ \\
\hline Ciprofloxacin & $8.00 \pm 2.54$ & $7.96 \pm 1.14$ & $8.05 \pm 1.60$ & $8.32 \pm 1.00$ & $7.43 \pm 0.45$ \\
\hline
\end{tabular}

Note: minimum inhibitory concentration (MIC) was measured with suitable dilutions (5-30 $\mu \mathrm{g} /$ well) and results were calculated using EZ-Fit Perrella Scientific Inc., Amherst, USA, software.

was presented by the three molecules, $9 \mathbf{e}, \mathbf{9 j}$, and $\mathbf{9 k}$ and their activity might be owed to the presence of ortho-substituted phenyl rings attached to nitrogen of acetamoyl linkage.

The alkyl substitution resulted in moderate to good activity against all the strains. Among aralkyl groups, the long aliphatic chain containing was moderate to good against all the strains. The molecules bearing ortho-substituted phenyl rings remained active against all the strains, also good to excellent and more efficient against the Gram-negative strains. The meta-substituted ones were also good against negative strain. The para-substituted phenyl rings presented varying activities but moderate ones.

\section{Conclusion}

The multistep synthesis was carried out to incorporate different functionalities with an aim to obtain more potent molecules. The most of the synthesized molecules remained 
efficient against all the strains specially Gram-negative bacteria. The most of the compounds were pharmacologically important including $9 \mathbf{e}, \mathbf{9 j}$, and $\mathbf{9 k}$. These molecules may be further modified to get more comparable or even better MIC results by more variation to the group linked to nitrogen of acetamoyl functionality. So the above listed compounds along with some others might be considered for drug designing program in search of new drug candidates.

\section{Competing Interests}

The authors declare that there are no competing interests regarding the publication of this paper.

\section{Acknowledgments}

Authors are much grateful to Higher Education Commission (HEC) of Pakistan regarding financial support for chemicals, solvents, and spectral analysis.

\section{References}

[1] A. Behrami and I. Krasniqi, "Antibacterial activity of coumarine derivatives synthesized from 4-chloro-chromen-2-one. The comparison with standard drug," Research Journal of Pharmaceutical, Biological and Chemical Sciences, vol. 3, no. 3, pp. 369375, 2012.

[2] T. Smyth, V. N. Ramachandran, and W. F. Smyth, "A study of the antimicrobial activity of selected naturally occurring and synthetic coumarins," International Journal of Antimicrobial Agents, vol. 33, no. 5, pp. 421-426, 2009.

[3] A. Kinza, M. K. Khosa, N. Jahan, and S. Nosheen, "Synthesis and applications of coumarin," Pakistan Journal of Pharmaceutical Sciences, vol. 23, no. 4, pp. 449-454, 2010.

[4] B. R. Dekić, N. S. Radulović, V. S. Dekić, R. D. Vukicević, and R. M. Palić, "Synthesis and antimicrobial activity of new 4heteroarylamino coumarin derivatives containing nitrogen and sulfur as heteroatoms," Molecules, vol. 15, no. 4, pp. 2246-2256, 2010.

[5] A. Sinhamahapatra, N. Sutradhar, S. Pahari, H. C. Bajaj, and A. B. Panda, "Mesoporous zirconium phosphate: an efficient catalyst for the synthesis of coumarin derivatives through Pechmann condensation reaction," Applied Catalysis A: General, vol. 394, no. 1-2, pp. 93-100, 2011.

[6] Y. Shi and C.-H. Zhou, "Synthesis and evaluation of a class of new coumarin triazole derivatives as potential antimicrobial agents," Bioorganic \& Medicinal Chemistry Letters, vol. 21, no. 3, pp. 956-960, 2011.

[7] N. N. Farshori, M. R. Banday, A. Ahmad, A. U. Khan, and A. Rauf, "7-Hydroxy-coumarin derivatives: synthesis, characterization and preliminary antimicrobial activities," Medicinal Chemistry Research, vol. 20, no. 5, pp. 535-541, 2011.

[8] M. Roussaki, C. A. Kontogiorgis, D. Hadjipavlou-Litina, S. Hamilakis, and A. Detsi, "A novel synthesis of 3-aryl coumarins and evaluation of their antioxidant and lipoxygenase inhibitory activity," Bioorganic \& Medicinal Chemistry Letters, vol. 20, no. 13, pp. 3889-3892, 2010.

[9] Y. Zhang, B. Zou, Z. Chen et al., "Synthesis and antioxidant activities of novel 4-Schiff base-7-benzyloxy- coumarin derivatives," Bioorganic and Medicinal Chemistry Letters, vol. 21, no. 22, pp. 6811-6815, 2011.
[10] A. Hasan, N. F. Thomas, and S. Gapil, "Synthesis, characterization and antifungal evaluation of 5-substituted-4-amino-1,2,4triazole-3-thioesters," Molecules, vol. 16, no. 2, pp. 1297-1309, 2011.

[11] P. J. Shirote and M. S. Bhatia, "Synthesis and goat pulmonary vasodilatory activity of some novel 1,3,4-oxadiazoles," Arabian Journal of Chemistry, vol. 4, no. 4, pp. 413-418, 2011.

[12] H. Gadegoni, S. Manda, and S. Rangu, "Synthesis and screening of some novel 2-[5-(Substituted phenyl)-[1,3,4] oxadiazol-2-yl]benzoxazoles as potential antimicrobial agents," Journal of the Korean Chemical Society, vol. 57, no. 2, pp. 221-226, 2013.

[13] R. Sawant and D. Kawade, "Synthesis and biological evaluation of some novel 2-phenyl benzimidazole-1-acetamide derivatives as potential anthelmintic agents," Acta Pharmaceutica, vol. 61, no. 3, pp. 353-361, 2011.

[14] G. Autore, A. Caruso, S. Marzocco et al., "Acetamide derivatives with antioxidant activity and potential anti-inflammatory activity," Molecules, vol. 15, no. 3, pp. 2028-2038, 2010.

[15] G. Ayhan-Kılcıgil, S. Gürkan, T. Çoban, E. D. Özdamar, and B. Can-Eke, "Synthesis and Evaluation of Antioxidant Properties of Novel 2-[2-(4-chlorophenyl) benzimidazole-1-yl]-N-(2arylmethylene amino) acetamides and 2-[2-(4-chlorophenyl) benzimidazole-1-yl]-N-(4-oxo-2-aryl-thiazolidine-3-yl) acetamides-I," Chemical Biology and Drug Design, vol. 79, no. 5, pp. 869-877, 2012.

[16] V. Kanagarajan, J. Thanusu, and M. Gopalakrishnan, "Synthesis and in vitro microbiological evaluation of an array of biolabile 2-morpholino- $N$-(4,6-diarylpyrimidin-2-yl)acetamides," European Journal of Medicinal Chemistry, vol. 45, no. 4, pp. 15831589, 2010.

[17] P. C. Sharma and S. Jain, "Synthesis and antibacterial activity of certain novel 1-cyclopropyl-6-flouro-1,4-dihydro-7-4-substituted-piperazin- 1-yl-4-oxoquinolin-3-carboxylates," Acta Pharmaceutica Sciencia, vol. 50, pp. 35-40, 2008.

[18] P. C. Sharma and S. Jain, "Synthesis and in-vitro antibacterial activity of some novel $N$-nicotinoyl-1-ethyl-6-fluoro-1,4dihydro-7-piperazin-1-yl-4-oxoquinoline-3-carboxylates," Acta Poloniae Pharmaceutica-Drug Research, vol. 65, no. 5, pp. 551556, 2008.

[19] Aziz-ur-Rehman, S. Rasool, M. A. Abbasi et al., "Synthesis, characterization and biological screening of some 4-Osubstituted derivatives of $\mathrm{N}$-(4-hydroxyphenyl)- $\mathrm{N}$-methyl-4methylbenzenesulfonamide," Asian Journal of Pharmaceutical and Biological Research, vol. 2, no. 2, pp. 100-105, 2012.

[20] S. Ghosh, P. Tiwari, S. Pandey et al., "Synthesis and evaluation of antitubercular activity of glycosyl thio- and sulfonyl acetamide derivatives," Bioorganic \& Medicinal Chemistry Letters, vol. 18, no. 14, pp. 4002-4005, 2008.

[21] M. Kaspady, V. K. Narayanaswamy, M. Raju, and G. K. Rao, "Synthesis, antibacterial activity of 2,4-disubstituted oxazoles and thiazoles as bioisosteres," Letters in Drug Design and Discovery, vol. 6, no. 1, pp. 21-28, 2009.

[22] C.-R. Yang, Y. Zhang, M. R. Jacob, S. I. Khan, Y.-J. Zhang, and X.-C. Li, "Antifungal activity of C-27 steroidal saponins," Antimicrobial Agents and Chemotherapy, vol. 50, no. 5, pp. 17101714, 2006.

[23] S. S. Bhattacharya, U. Das, and B. K. Choudhury, "Occurrence \& antibiogram of Salmonella typhi \& S. paratyphi A isolated from Rourkela, Orissa," Indian Journal of Medicinal Research, vol. 133, no. 4, pp. 431-433, 2011. 
[24] R. L. Vogt and L. Dippold, "Escherichia coli O157:H7 outbreak associated with consumption of ground beef, June-July 2002," Public Health Reports, vol. 120, no. 2, pp. 174-178, 2005.

[25] T. Pressler, C. Bohmova, S. Conway et al., "Chronic Pseudomonas aeruginosa infection definition: EuroCareCF Working Group report," Journal of Cystic Fibrosis, vol. 10, supplement 2, pp. S75-S78, 2011.

[26] V. Barbe, S. Cruveiller, F. Kunst et al., "From a consortium sequence to a unified sequence: the Bacillus subtilis 168 reference genome a decade later," Microbiology, vol. 155, no. 6, pp. 1758-1775, 2009.

[27] L. G. Harris, S. J. Foster, R. G. Richards, P. Lambert, D. Stickler, and A. Eley, "An introduction to Staphylococcus aureus, and techniques for identifyingand quantifying $S$. aureus adhesins in relation to adhesion to biomaterials: review," European Cells and Materials, vol. 4, pp. 39-60, 2002. 

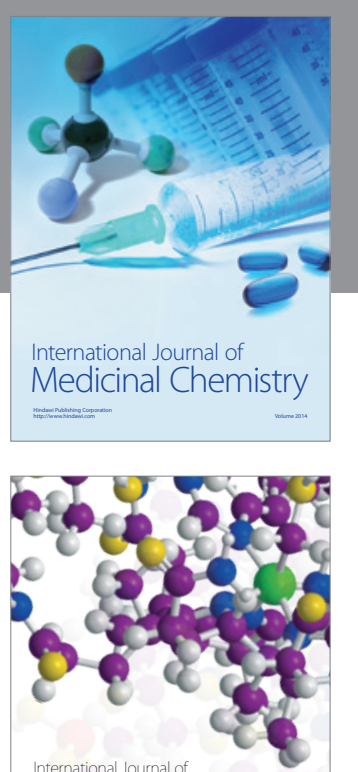

Carbohydrate Chemistry

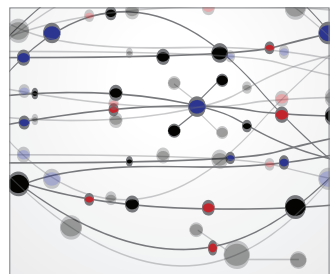

The Scientific World Journal
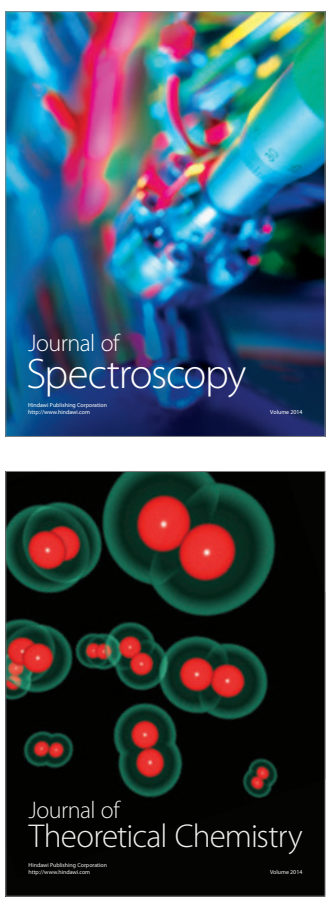
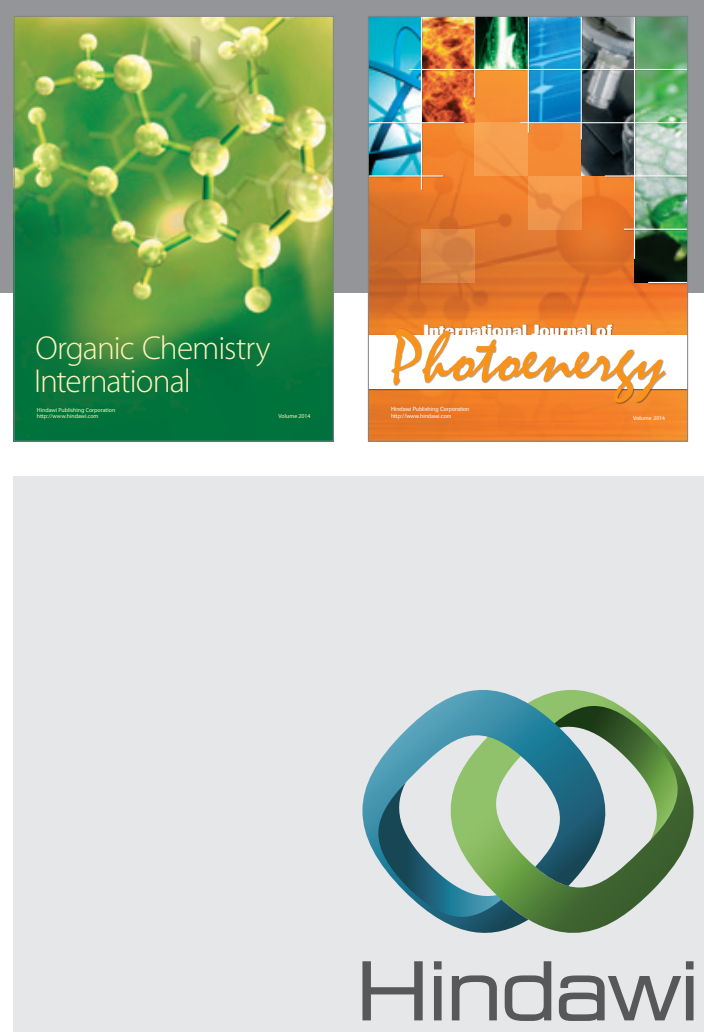

Submit your manuscripts at

http://www.hindawi.com

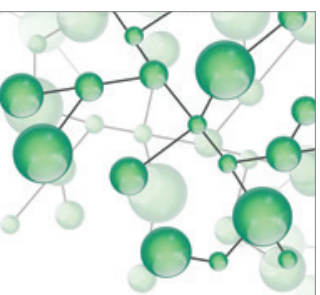

International Journal of

Inorganic Chemistry

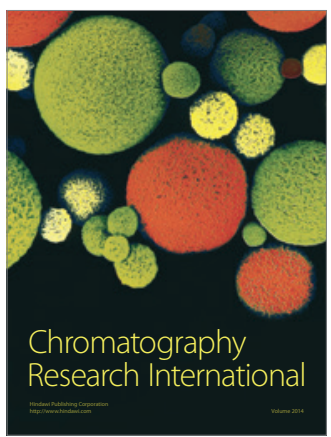

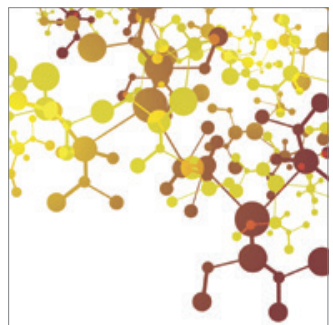

Applied Chemistry
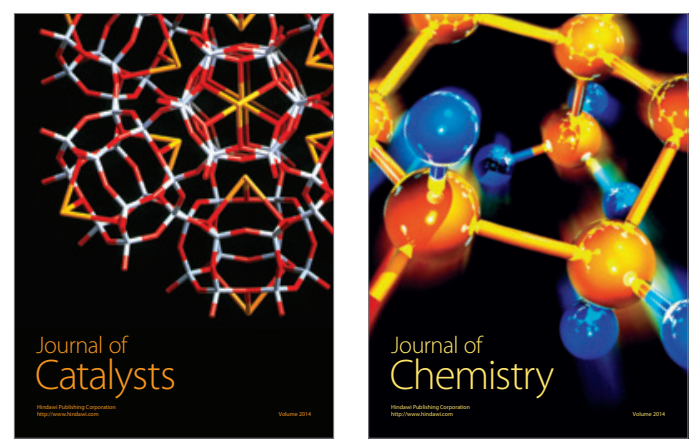
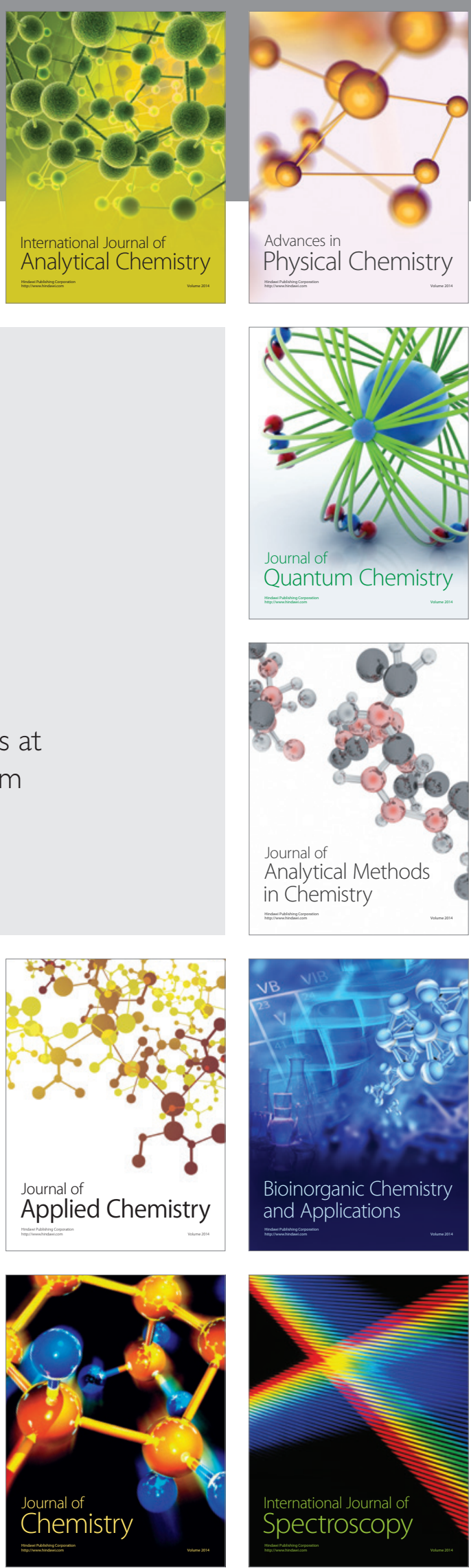